\title{
Concurrent X-ray, near-infrared, sub-millimeter, and GeV gamma-ray observations of Sagittarius $A^{*}$
}

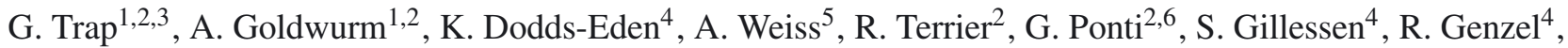 \\ P. Ferrando ${ }^{1,2}$, G. Bélanger ${ }^{7}$, Y. Clénet ${ }^{8}$, D. Rouan ${ }^{8}$, P. Predehl ${ }^{4}$, R. Capelli ${ }^{4}$, F. Melia ${ }^{9}$, and F. Yusef-Zadeh ${ }^{10}$ \\ 1 Service d'Astrophysique (SAp), IRFU, DSM, CEA-Saclay, 91191 Gif-sur-Yvette, France \\ e-mail: trap@apc.univ-paris7.fr \\ 2 AstroParticule \& Cosmologie (APC), Université Paris VII, CNRS, CEA, Observatoire de Paris, 75205 Paris, France \\ 3 Département de Physique, Palais de la découverte, Universcience, 75008 Paris, France \\ ${ }^{4}$ Max-Planck-Institut für Extraterrestrische Physik (MPE), 85748 Garching, Germany \\ 5 Max-Planck-Institut für Radioastronomie (MPIfR), 53121 Bonn, Germany \\ ${ }^{6}$ School of Physics and Astronomy, University of Southhampton, Highfield, Southhampton, SO17 1BJ, UK \\ 7 European Space Agency (ESA), ESAC, PO Box 778, Villanueva de la Canada, 28691 Madrid, Spain \\ 8 Laboratoire d'Études Spatiales et d'Instrumentation en Astrophysique (LESIA), Observatoire de Paris, 92195 Meudon, France \\ 9 Department of Physics and Steward Observatory, University of Arizona, Tucson, Arizona 85721, USA \\ 10 Department of Physics and Astronomy, Northwestern University, Evanston, Illinois 60208, USA
}

Received 4 June 2010 / Accepted 5 February 2011

\section{ABSTRACT}

\begin{abstract}
Aims. The radiative counterpart of the supermassive black hole at the Galactic center (GC), Sgr A ${ }^{\star}$, is subject to frequent flares that are visible simultaneously in X-rays and the near-infrared (NIR). Often, enhanced radio variability from centimeter to sub-millimeter wavelengths is observed to follow these X-ray/NIR eruptions. We present here a multi-wavelength campaign carried out in April 2009, with the aim of characterizing this broadband flaring activity.

Methods. Concurrent data from the XMM-Newton/EPIC (2-10 keV), VLT/NACO $(2.1 \mu \mathrm{m}, 3.8 \mu \mathrm{m})$, APEX/LABOCA (870 $\mu \mathrm{m})$, and Fermi/LAT $(0.1-200 \mathrm{GeV})$ instruments are employed to derive light curves and spectral energy distributions of new flares from $\operatorname{Sgr} A^{\star}$.

Results. We detected two relatively bright NIR flares, both associated with weak X-ray activity, one of which was followed by a strong sub-mm outburst 200 min later. Photometric spectral information on a NIR flare was obtained for the first time with NACO, giving a power-law photon index $\alpha=-0.4 \pm 0.3\left(F_{v} \propto v^{\alpha}\right)$. The first attempt to detect flaring activity from the Fermi GC source 1FGL J1745.6-2900 is also reported. We model NIR, X-ray, and sub-mm flares in the context of non-thermal emission processes. We find that the simplest scenario involving a single expanding plasmoid releasing synchrotron NIR/sub-mm and synchrotron selfCompton X-ray radiation is inadequate to reproduce the data, but we offer suggestions to reconcile the basic elements of the theory and the observations.
\end{abstract}

Key words. Galaxy: center - black hole physics - radiation mechanisms: non-thermal - X-rays: general - infrared: general submillimeter: general

\section{Introduction}

The tracing of stellar orbits in the heart of the Milky Way since the early 1990's has definitely established the presence of a central high concentration of mass, $\sim 4 \times 10^{6} M_{\odot}$, which is confined to a region no bigger than the Solar System (Ghez et al. 2008; Gillessen et al. 2009). This is the best evidence to date for the existence of a supermassive black hole at the Galactic center (GC, see Melia 2007, for a comprehensive review). The relative proximity of this black hole to us $(8 \mathrm{kpc}$, Reid et al. 2009) has enabled thorough observations of its weak pointlike radiative manisfestation, Sagittarius $A^{\star}\left(\operatorname{Sgr} A^{\star}\right)$, accross the entire electromagnetic spectrum. In spite of a high interstellar extinction, wich precludes detections at optical and ultraviolet wavelengths, $\mathrm{Sgr} \mathrm{A}^{\star}$ has now been positively detected in radio (from cm to sub-mm wavelengths, Balick \& Brown 1974; Zylka \& Mezger 1988), near-infrared (NIR, Genzel et al. 2003), and X-rays (Baganoff et al. 2003). In the vast $\gamma$-ray domain, point-like sources coincident with the position of $\mathrm{Sgr} \mathrm{A}^{\star}$ have been found at the $10 \mathrm{keV}$ (IGR J17456-2901, Bélanger et al. 2004), GeV (1FGL J1745.6-2900, Abdo et al. 2010), and TeV (HESS J1745-290, Aharonian et al. 2004) levels, though their association with the supermassive black hole is less evident.

One striking feature of $\operatorname{Sgr~}^{\star}$ is its temporal variability. Albeit much more quiet than typical active galactic nuclei (AGNs), Sgr A ${ }^{\star}$ displays rapid flares from time to time in $\mathrm{X}$-rays and NIR, which temporarily enhance its luminosity by factors up to $\sim 160$ on timescales of $1-2 \mathrm{~h}$. X-ray flares have been discovered at a rate of about one event per day by the Chandra (Baganoff et al. 2001) and XMM-Newton (Goldwurm et al. 2003) satellites, while NIR flares seem to recur more frequently (2-6 events per day) as observed by the VLT (Genzel et al. 2003), the Keck (Ghez et al. 2004), the HST (Yusef-Zadeh et al. 2006a), and Subaru (Nishiyama et al. 2009).

These flares usually present short timescale variations that point to an emitting region with a size on the order of $10 R_{\mathrm{S}}$ or less, where $R_{\mathrm{S}}$ is the Schwarzschild radius of the black hole. They have thus offered new probes into the central engine and 
received considerable attention of extensive multi-wavelength campaigns since their discoveries. On the one hand, these campaigns revealed that all X-ray flares seem to have a simultaneous NIR counterpart (Eckart et al. 2004, 2006a, 2008a; Hornstein et al. 2007; Yusef-Zadeh et al. 2006a, 2009; Dodds-Eden et al. 2009), while not all NIR flares have detectable X-ray counterparts (Hornstein et al. 2007). Considering also the linear polarization properties of NIR flares (e.g., Eckart et al. 2006b), a general consensus emerged in which the origin of NIR flares was ascribed to synchrotron radiation of transiently accelerated electrons and that of X-ray flares to the corresponding inverse Compton emission, especially synchrotron self-Compton (SSC) (e.g., Liu et al. 2006a,b). However, the recent study of an exceptionally bright X-ray/NIR flare detected on 2007 April 4, challenged the SSC model (Dodds-Eden et al. 2009; Trap et al. 2010). This proved the necessity to measure contemporaneous spectra (with good statistics) in infrared and in X-rays to constrain the overall spectral energy distribution (SED) of the flares. Yet, past attempts to obtain NIR spectral information on $\mathrm{Sgr}^{\star}{ }^{\star}$ have given various and sometimes conflicting results (e.g., Gillessen et al. 2006; Hornstein et al. 2007).

On the other hand, broadband campaigns found indications that contemporaneous X-ray/NIR flares could be accompanied by delayed radio activity (Yusef-Zadeh et al. 2006a; Eckart et al. 2006a). This was interpreted as a sign of expanding plasmoids in the vicinity of the supermassive black hole, which release optically thin and thick synchrotron emission whose peak evolves with frequency (van der Laan 1966). Recently, Marrone et al. (2008) and Yusef-Zadeh et al. (2008) clearly detected an increase in the $\mathrm{mm}$ and sub-mm emission, using the Sub-Millimeter Array (SMA) and the Caltech Sub-millimeter Observatory (CSO) respectively, following an X-ray/NIR flare detected by Chandra and the Keck observatories. In the frame of an SSC/expanding blob model, Marrone et al. (2008) argued that there should be an observable correlation between the X-ray-to-NIR peak ratio and the sub-mm time delay, which could help test this picture.

We present here a new X-ray, NIR, and sub-mm campaign involving the X-ray Multi-Mirror (XMM)-Newton satellite, the Very Large Telescope (VLT), and the Atacama Pathfinder EXperiment (APEX) observatory, respectively. The data obtained enable us to investigate the aforementioned areas of debate by measuring Sgr $A^{\star}$ 's time variability and spectrum across a broad range of wavelengths. Concurrent public $\mathrm{GeV} \gamma$-ray data from the Fermi satellite are also examined. Additional radio data will be reported elsewhere.

This paper is organized as follows. In Sect. 2 we give brief summaries of the data acquisition and reduction of each telescope. In Sect. 3 we describe the respective light curves of $\mathrm{Sgr} \mathrm{A}^{\star}$, identify new flares, and compare their properties with past detections. Section 4 is dedicated to the NIR color measurements performed on one particular flare, while Sect. 5 discusses possible phenomenological interpretations of the light curves and SEDs of all the newly detected flares.

\section{Observations and data analysis}

We observed the GC in early April 2009 in the context of an extensive multi-wavelength campaign that was initiated by an $X M M-N e w t o n / V L T$ joint program and followed by an APEX monitoring scheduled accordingly. A detailed observation journal for this set of instruments is given in Table 1. Fermi data were also recorded at the epoch of this campaign as part of the
Table 1. Observation log.

\begin{tabular}{|c|c|c|c|c|}
\hline Orbit & OвsID & $\begin{array}{c}\text { START DATE } \\
{[\text { UTC] }}\end{array}$ & $\begin{array}{l}\text { Exp. } \\
{[\mathrm{ks}]}\end{array}$ & BAND \\
\hline & & XMM-Newton/PN & & \\
\hline 1705 & 554750401 & 2009 Apr. 1, 01:18:30 & 37.8 & $2-10 \mathrm{keV}$ \\
\hline 1706 & 554750501 & 2009 Apr. 3, 01:56:31 & 42.0 & $2-10 \mathrm{keV}$ \\
\hline \multirow[t]{2}{*}{1707} & 554750601 & 2009 Apr. 5, 03:53:34 & 32.4 & $2-10 \mathrm{keV}$ \\
\hline & & VLT/NACO & & \\
\hline & 082.B-0952A & 2009 Apr. 1, 06:46:59 & 11.5 & $3.8 \mu \mathrm{m}\left(L^{\prime}\right)$ \\
\hline \multirow{2}{*}{$\begin{array}{l}\ldots \\
\ldots \ldots\end{array}$} & 082.B-0952A & 2009 Apr. 3, 07:25:41 & 9.7 & $2.1 \mu \mathrm{m}\left(K_{\mathrm{s}}\right)$ \\
\hline & 082.B-0952A & 2009 Apr. 3, 07:28:42 & 9.7 & $3.8 \mu \mathrm{m}\left(L^{\prime}\right)$ \\
\hline \multirow[t]{2}{*}{$\ldots \ldots$} & 082.B-0952A & 2009 Apr. 5, 08:44:10 & 6.6 & $3.8 \mu \mathrm{m}\left(L^{\prime}\right)$ \\
\hline & & APEX/LABOCA & & \\
\hline & 183.B-0100A & 2009 Apr. 1, 05:31:43 & 30.7 & $870 \mu \mathrm{m}$ \\
\hline & 183.B-0100A & 2009 Apr. 3, 11:07:43 & 8.2 & $870 \mu \mathrm{m}$ \\
\hline$\ldots$ & 183.B-0100A & 2009 Apr. 5, 06:40:10 & 25.6 & $870 \mu \mathrm{m}$ \\
\hline
\end{tabular}

ongoing monitoring of the $\mathrm{GeV} \gamma$-ray sky. We present these data and their respective treatments below.

\subsection{XMM-Newton (2-10 keV)}

The X-ray satellite XMM-Newton (Jansen et al. 2001) pointed toward the GC on three occasions between April 1 and April 5 $(\sim 3 \times 40 \mathrm{ks})$. In the following, we will consider the data recorded with the Medium filter by the European Photon Imaging Camera (EPIC), i.e. the PN (Strüder et al. 2001) and the MOS 1-2 cameras (Turner et al. 2001) instruments, all operated in Prime Full Window mode. The data were reduced and analyzed with the Science Analysis Software (SAS) package (version 8.0.0) and the latest calibration files available at the time of the analysis. They were not contaminated by soft proton flares from solar activity. Starting with the original observation data files, event lists were generated through the emchain and epchain routines, and filtered with PATTERN $<=4$ or 12 (for PN and MOS respectively), to ensure the selection of X-rays and rejection of cosmic-rays. In order to prevent the use of X-ray photons that fell on bad pixels, we also applied the \#XMMEA_EP and \#XMMEA_EM filters on the event lists. Finally, artefacts from the calibrated and concatenated datasets and events near CCD gaps were discarded by setting FLAG $==\theta$ as selection criterion. The high interstellar absorption toward the GC $\left(N_{\mathrm{H}} \approx 10^{23} \mathrm{~cm}^{-2}\right)$ prevents the use of photons below $\sim 2 \mathrm{keV}$, so that the following count rates, fluxes, and luminosities will refer to the $2-10 \mathrm{keV}$ band.

The image obtained by the PN detector and reproduced in Fig. 1 (middle panel) reveals the usual constituents of the Galactic nucleus visible via XMM-Newton: extended thermal emission from the shell of the supernova remnant Sgr A East (Sakano et al. 2004) and point-like emission conincident with the position of $\operatorname{Sgr} \mathrm{A}^{\star}$. In addition, these observations allowed us to locate another point source at only $\sim 27^{\prime \prime}$ from Sgr $A^{\star}$, which we identify with the weak transient CXOGC J174538.0-290022 $\left(\sim 2 \times 10^{34} \mathrm{erg} \mathrm{s}^{-1}\right.$ at $8 \mathrm{kpc}$, Ponti et al. 2009).

To search for time variability of $\mathrm{Sgr} \mathrm{A}^{\star}$, we first fitted the positions of the point sources by running the edetect_chain procedure. A light curve of Sgr $A^{\star}$ was then extracted from a $10^{\prime \prime}$ radius circular region centered on the fitted position in each EPIC detector. These light curves were background-subtracted, with the background regions selected following the prescriptions given in Kirsch (2005) and the counts scaled to the same extraction area as $\mathrm{Sgr} \mathrm{A}^{\star}$. Finally, the $2-10 \mathrm{keV}$ backgroundsubtracted PN, MOS 1, and MOS 2 light curves were summed and rebinned with a sampling of $600 \mathrm{~s}$ for the first and third observations, and $400 \mathrm{~s}$ for the second. As resolved by previous 


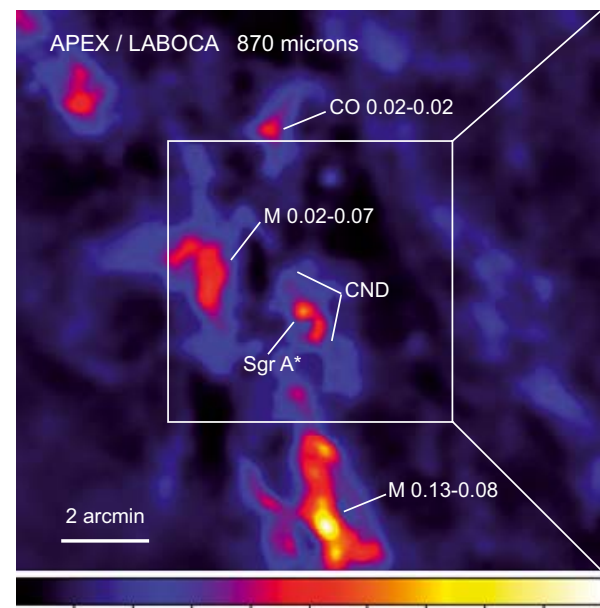

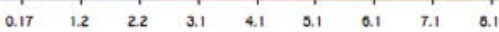
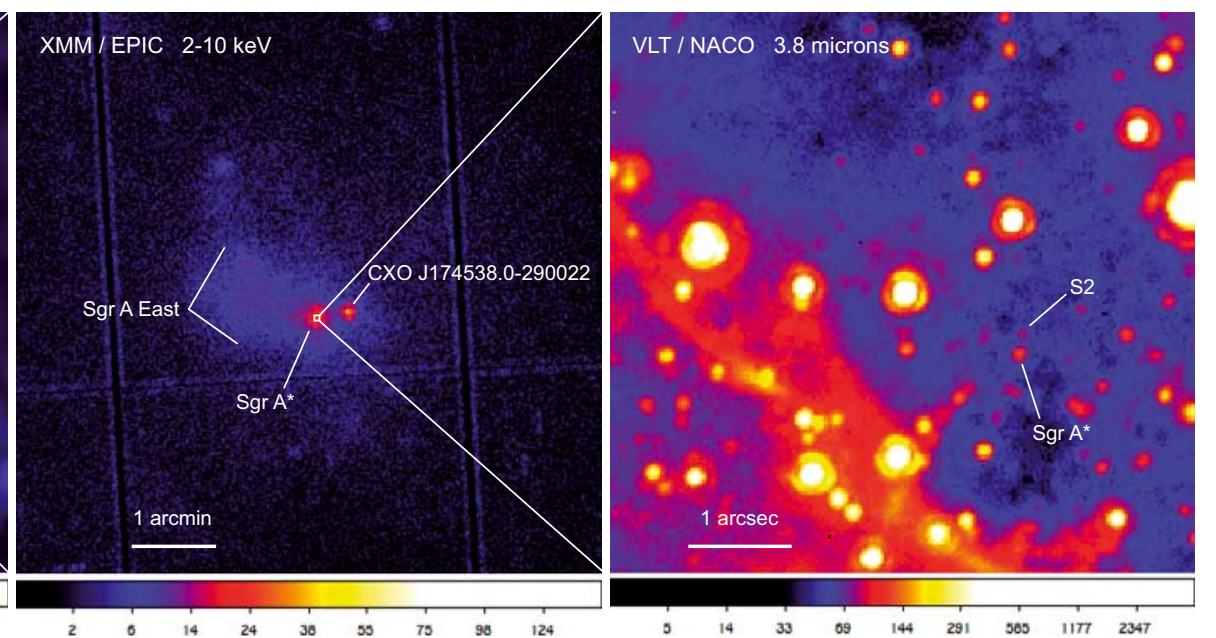

Fig. 1. Multi-wavelength images of the Galactic center in early April 2009. North is to the top, East to the left, and the Galactic plane runs from upper left to bottom right. The sub-mm and X-ray images (left and middle panels) are summed over one or several observations, whereas the $L^{\prime}$-band NIR image (right panel) corresponds to the summit of Flare A. Color scales are given in units of Jy beam ${ }^{-1}$, counts pixel ${ }^{-1}$, and ADU, from left to right, respectively.

Chandra deep observations, the persistent X-ray emission from a $10^{\prime \prime}$ radius region centered on $\mathrm{Sgr} \mathrm{A}^{\star}$ not only contains the $\mathrm{X}$-ray counterpart of $\mathrm{Sgr} \mathrm{A}^{\star}$, but also includes persistent compact sources such as the star group IRS 13 (Baganoff et al. 2003) and the pulsar wind nebula candidate G 359.95-0.04 (Wang et al. 2006), possible transient point sources (Muno et al. 2005), and a diffuse component (Muno et al. 2003).

\section{2. $\operatorname{VLT}(2.1 \mu \mathrm{m}$ and $3.8 \mu \mathrm{m})$}

The three X-ray observations described in the last paragraph were partly covered by NIR observations with the NACO instrument NAOS (Nasmyth Adaptive Optics System, Lenzen et al. 2003) + CONICA (COudé Near Infrared CAmera, Rousset et al. 2003), mounted on the fourth unit telescope, Yepun, at ESO's VLT in Paranal, Chile. These observations were divided into three half nights (2009 April 1, 3, and 5), which all started in the middle of the night and were extended as long as possible until sunrise.

We acquired data solely in the $L^{\prime}$-band $(3.8 \mu \mathrm{m})$ during the first and third nights, while during the second night, the atmospheric conditions were favorable to also collect images in the $K$-short $\left(K_{\mathrm{s}}\right)$ band at $2.1 \mu \mathrm{m}$. During this last night, we attempted a novel filter-switching technique with NACO to study the color of $\mathrm{Sgr} \mathrm{A}^{\star}$ from broadband quasi-simultaneous NIR measurements. Up to now, it has only been possible to investigate the spectrum of $\mathrm{Sgr}^{\star}$ at the VLT through the integral field spectrograph SINFONI over relatively narrow bands: the $H+K$-band $(\sim 1.7-2.4 \mu \mathrm{m}$, Eisenhauer et al. 2005) and the $K$-band ( 2.0$2.4 \mu \mathrm{m}$, Gillessen et al. 2006). Using NACO, we obtained for the first time spectral data over a wider wavelength range and with better Strehl ratios by constantly cycling back and forth between $K_{\mathrm{s}}$ and $L^{\prime}$ photometric filters throughout the night. We carried out successive full switches (switching $K_{\mathrm{s}}$ - to $L^{\prime}$-band, acquiring two $L^{\prime}$-band images, switching $L^{\prime}$ - to $K_{\mathrm{s}}$-band and acquiring two $K_{\mathrm{s}}$-band images) on a timescale of only $\sim 5-5.5 \mathrm{~min}$. All the data examined here were collected with the supergiant IRS 7 as natural guide star and the infrared wavefront sensor to close the adaptive optics loop of NAOS. Images were individually sky-subtracted, flat-fielded, and corrected for dead/hot pixels (see Fig. 1, right panel).
In Figs. 3, 4, and 5 we display the light curves of $\mathrm{Sgr} \mathrm{A}^{\star}$ corrected for extinction with the usual values $A_{K_{\mathrm{s}}}=2.8 \mathrm{mag}$ and $A_{L^{\prime}}=1.8 \mathrm{mag}$, to allow a comparison with previous works (e.g., Genzel et al. 2003; Dodds-Eden et al. 2009). They were obtained via aperture photometry and calibrated with the fluxes of nearby stars of known and stable brightness: all fluxes were scaled so that the median flux of the relatively isolated star S 65 always corresponds to an apparent $K_{\mathrm{s}}$ magnitude of $13.7 \mathrm{mag}$ (Gillessen et al. 2009) and the median flux of IRS $16 \mathrm{NW}$ to an apparent $L^{\prime}$ magnitude of $8.38 \mathrm{mag}$ (Viehmann et al. 2005). More details on the second night light curves are given in Sect. 4. The duration of the third night light curve is reduced compared with the other two owing to a technical problem with the telescope. We stress that at the time of these observations, $\mathrm{Sgr} \mathrm{A}^{\star}$ was confused with the star S 17, whose contribution to the light curves is expected to be a constant value of $\sim 5.4 \mathrm{mJy}$ and $\sim 3.5 \mathrm{mJy}$ in the $K_{\mathrm{s}}-$ and $L^{\prime}$ band, respectively (see S 17 magnitudes reported in Ghez et al. 2005 , corrected with the aforementioned values of $A_{\lambda}$ ).

\section{3. $\operatorname{APEX}(870 \mu \mathrm{m})$}

In parallel to the X-ray and NIR surveys, a total of $\sim 20 \mathrm{~h}$ of sub-mm GC data were taken at $870 \mu \mathrm{m}(345 \mathrm{GHz})$. We used the Large APEX bolometer camera (LABOCA, Siringo et al. 2009), on the APEX telescope (Güsten et al. 2006) at Llano Chajnantor, Chile. LABOCA is an array of 295 composite bolometers operating in the $345 \mathrm{GHz}$ transmission window. The angular resolution (FWHM) of each beam is $\sim 19^{\prime \prime}$ for a bandwidth of $\sim 60 \mathrm{GHz}$. LABOCA's layout leads to a double-beam spaced distribution of the individual beams in a hexagonal configuration over a 11.4' field of view (FOV).

The observations performed on the April 1 and 5 spanned $\sim 8.5$ and $\sim 7.5 \mathrm{~h}$, respectively, with an interruption of $1 \mathrm{~h}$ and 15 min between UT 8:45 and 10:00. During this time interval, the GC passed above the telescope's elevation limit of $\sim 80^{\circ}$. Observations on April 3 only covered the period after this GC transit (UT 11:00-13:30). Note that the APEX and VLT sites have basically the same longitude, but, contrary to the VLT, APEX could continue observing past sunrise, till the elevation of the GC was too low to allow for reliable monitoring purposes (elevation between $30^{\circ}$ and $40^{\circ}$, depending on weather conditions). 
The precipitable water vapor during the three runs was $\sim 2, \sim 0.9$, and $0.2-0.4 \mathrm{~mm}$, respectively.

The GC was observed using "on the fly" maps with scanning angles of $-15^{\circ}, 0^{\circ}$, or $+15^{\circ}$ with respect to the orthogonal direction across the Galactic plane. The maps were built with a scanning speed of $2 \operatorname{arcmin~s}^{-1}$ and steps orthogonal to the scanning direction of $30^{\prime \prime}$. The total integration time of each map was 315 s. Each GC map was followed by observations of G 10.62 and G 5.89, standard LABOCA secondary calibrators. The atmospheric zenith opacity was determined via skydips every 1$2 \mathrm{~h}$. With this observing setup, Sgr A ${ }^{\star}$ flux was monitored every $\sim 8$ min.

The data were reduced using the BoA software package. Reduction steps on the bolometer time series include temperature-drift correction based on two "blind" bolometers, flat-fielding, calibration, opacity correction, correlated noise removal on the full array as well as on groups of bolometers related by the wiring and in the electronics, flagging of bad bolometers, and finally de-spiking. Each reduced scan was then gridded into a spatial intensity and weighting map. These reduction steps were applied to the GC and both calibrators scans. Flux calibration was achieved using G 10.62, which has a known flux density of $33.4 \pm 2.0 \mathrm{Jy}$ for LABOCA (Siringo et al. 2009). The absolute calibration accuracy is roughly $10 \%$. To infer the relative calibration error, we applied the calibration curve of G 10.62 to G5.89 and determined the dispersion of the measured G5.89 fluxes over the observing period. This yielded a relative calibration accuracy of $\sim 3 \%$.

For the determination of Sgr $\mathrm{A}^{\star}$ 's light curve we first generated a model of the sub-mm emission of the region by co-adding all calibrated and pointing-drift corrected GC maps. In this high signal-to-noise map (see Fig. 1, left panel), we note the presence of several molecular clouds in accordance with previous submm GC surveys (Pierce-Price et al. 2000), and the point source Sgr $A^{\star}$, which was fitted with a Gaussian and later subtracted from the co-added map. We finally re-reduced all GC scans and subtracted the model signal from the time series of each bolometer. The resulting maps only contain the point source Sgr A ${ }^{\star}$. The light curves were eventually constructed by fitting a Gaussian to each individual map (see Figs. 3-5). The error bars were estimated through the RMS of the calibrator G5.89 ( 2.7\%). $\mathrm{Sgr}^{\star}$ does not suffer from any significant interstellar extinction at $870 \mu \mathrm{m}$.

\subsection{Fermi $(0.1-200 \mathrm{GeV})$}

The Fermi space observatory carries the Large Area Telescope (LAT, Atwood et al. 2009), a high-energy $\gamma$-ray pair-conversion telescope covering the $\sim 30 \mathrm{MeV}$ to $\sim 300 \mathrm{GeV}$ band with a wide FOV (2.4 sr). It operates almost as an all-sky monitor and can provide regular $\sim 50 \mathrm{~min}$ uninterrupted exposures on the GC. Using the public Fermi data archive, we explored the periods covered by the aforementioned observations of April 2009, and searched for a potential $\gamma$-ray flaring activity correlated with variability in other spectral domains.

The LAT data were analyzed with the Fermi science tools and following the analysis threads distributed by the Fermi Science Support Center. We created event cubes with the gtselect routine by selecting only $\gamma$-ray photons ("diffuse" class events, No. 3). We then used gtmktime and the "spacecraft" file, which keeps track of the satellite's attitude as a function of time, to perform good time interval selections. A zenithangle cut was thereby applied $\left(z_{\max }=105^{\circ}\right)$, rejecting $\gamma$-rays emanating from Earth albedo. The light curves were derived

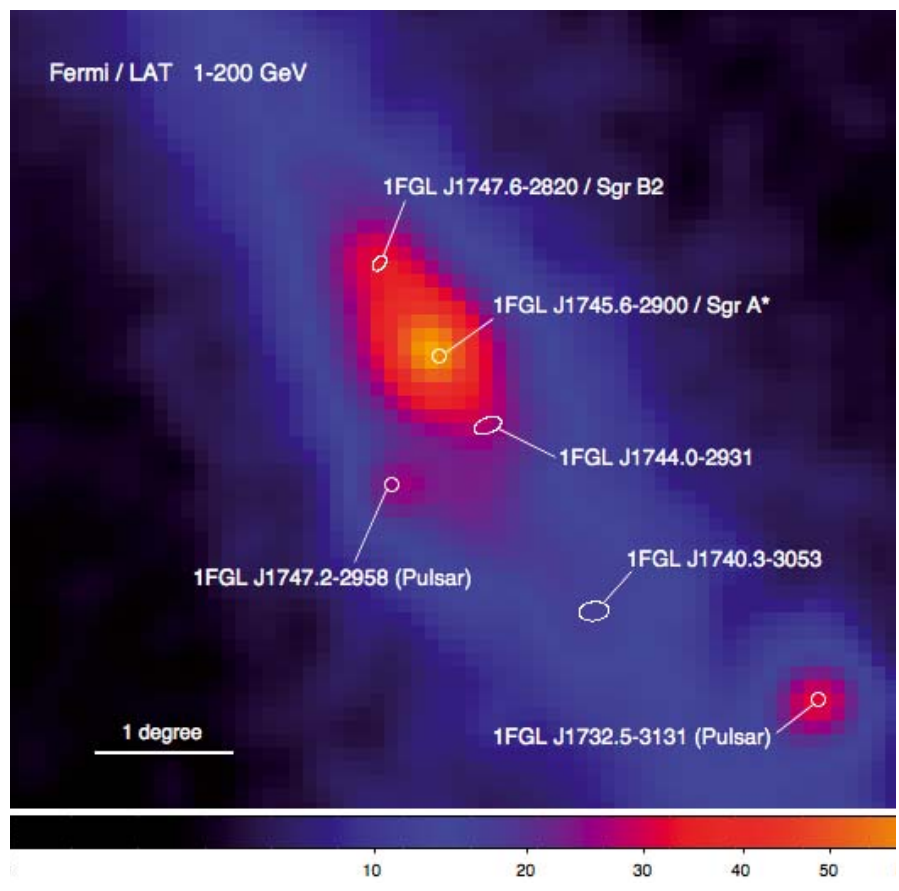

Fig. 2. Map of the Galactic center built with $1-200 \mathrm{GeV} \gamma$-ray photons collected by the LAT instrument during the first year of the Fermi mission (Aug. 2008/Aug. 2009). North is to the top, East to the left, and the Galactic plane runs from upper left to bottom right. Each pixel has a size of $0.1^{\circ}$ and the image was smoothed with a Gaussian kernel of $0.15^{\circ}$ radius. The color bar is expressed in counts and the positions of the point sources were taken from Abdo et al. (2010).

via aperture photometry (gtbin) with a circular extraction region of radius $1^{\circ}$, centered on the position of Sgr $A^{\star}$. The fluxes thus measured include the diffuse $\gamma$-ray emission associated with cosmic-ray interactions in GC molecular clouds. The number of counts in each bin, $n$, which are governed by Poissonian statistics, $1 \sigma$ (84\% confidence level) error bars were approximated by $1+\sqrt{n+0.75}$ (Gehrels 1986). Finally, we computed the exposures of each time bin in $\mathrm{s}^{-1} \mathrm{~cm}^{-2}$ with gtexposure. The resulting $0.1-200 \mathrm{GeV}$ light curves are presented in the top panels of Figs. 3-5 with a time bin of $600 \mathrm{~s}$ (10 min).

For illustration purposes, we produced an average LAT image of the GC (Fig. 2) using all the data from the first year of full sky survey, from August 2008 to August 2009. Only highenergy photons from the $1-200 \mathrm{GeV}$ band were considered to optimize the single photon angular resolution $\left(<0.6^{\circ}\right.$ at energies $>1 \mathrm{GeV})$.

\section{Light curves and flares}

In this section we discuss the properties of the multi-wavelength light curves and compare them with the relevant literature.

\subsection{NIR}

The first night of observations was marked by a strong NIR flare detected in the $L^{\prime}$-band between $440-515$ min past midnight (Fig. 3), hereafter referred to as "Flare A". It was brighter than the star S 2 (see the pale red dot north of Sgr A ${ }^{\star}$ in Fig. 1, right panel) and peaked at $19 \pm 1 \mathrm{mJy}$ (background subtracted), which makes it the second brightest $L^{\prime}$ flare covered in X-rays and one of the few powerful NIR flares so far detected that are on the 
G. Trap et al.: Concurrent X-ray, NIR, Sub-mm, and GeV $\gamma$-ray observations of Sgr A*

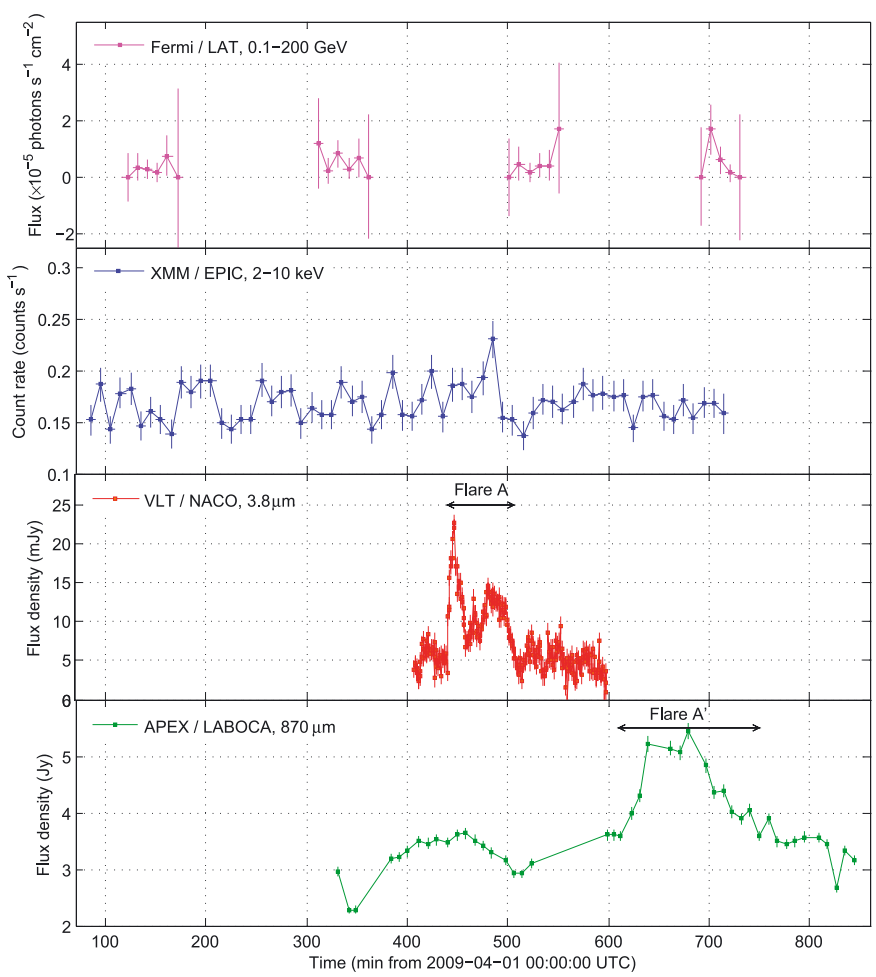

Fig. 3. Light curves of $\mathrm{Sgr}^{\star}{ }^{\star}$ and its associated backgrounds (see text for details) during the first observation.

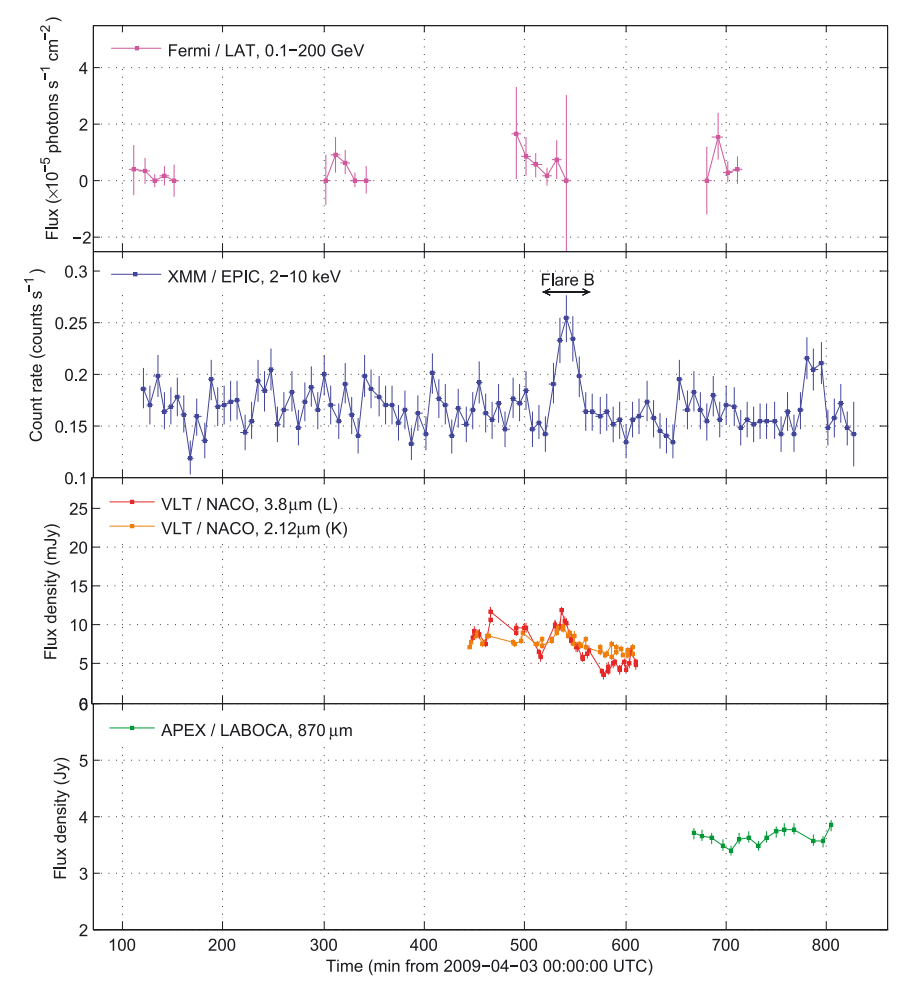

Fig. 4. Same as Fig. 3 for the second observation.

order of $20 \mathrm{mJy}$ (in all NIR bands considered) or beyond (DoddsEden et al. 2009; Eckart et al. 2008a,b; Meyer et al. 2006b, 2007). Flare A has a remarkable complex non-symmetrical morphology, which is characterized by a very sharp rise (a continuous gain of $\sim 19 \mathrm{mJy}$ in $\sim 7 \mathrm{~min}$ ) followed by a slower and non-monotonic decay. This rise time is one of the fastest ever reported and is actually fairly unusual among strong NIR flares.

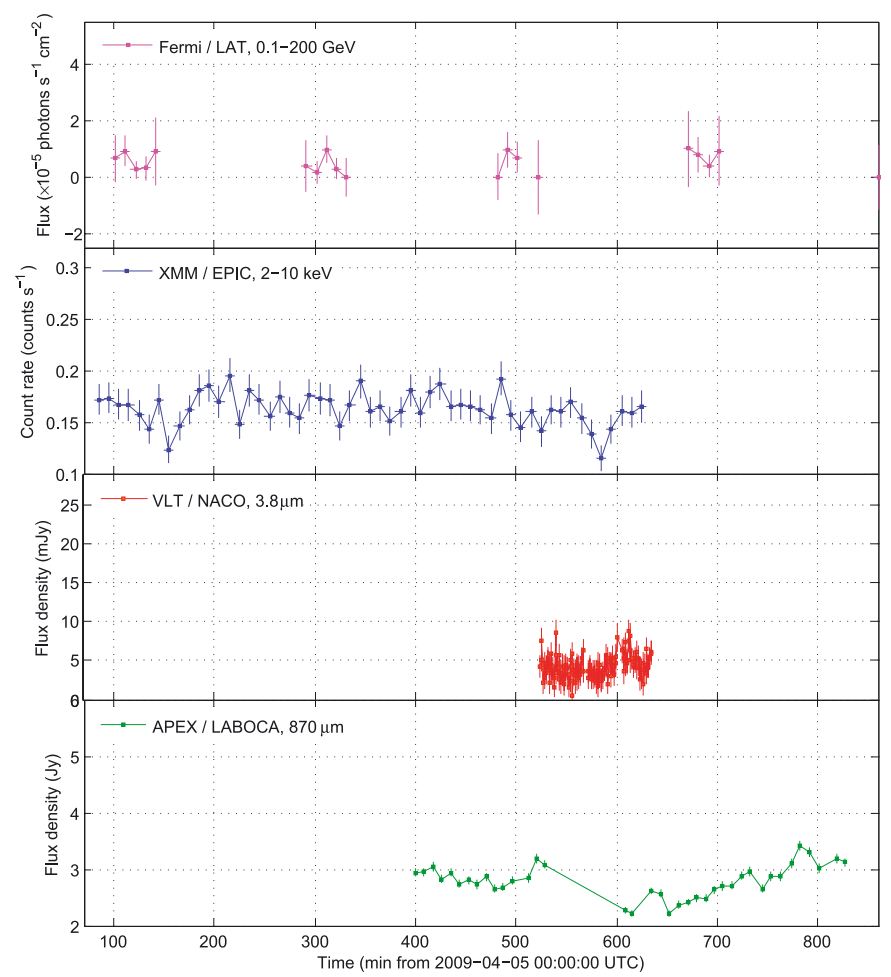

Fig. 5. Same as Fig. 3 for the third observation.

It hence places a stringent upper limit on the size of the overall flare emitting region of $\sim 10 R_{\mathrm{S}}$ ( or $\sim 1 \mathrm{AU}$ ), with a causality argument $(R \leq c \Delta t)$ and considering a mass of $4 \times 10^{6} M_{\odot}$ for the black hole.

During the second night the time resolution of the light curves was not as good as during the other nights, given the continuous cycling through the $K$ and $L$ filters, which makes the identification of flares more difficult. Sgr A ${ }^{\star}$ seems to have been continuously variable in both bands at a significant level (see Fig. 7, top panel, and Sect. 4), at least during the first two thirds of the observation. Our X-ray coverage allows us to clearly isolate a new eruption - Flare B - between $\sim 520$ and $\sim 560$ min after 2009 April 3, 00:00:00 (UTC) though (see Sects. 3.2 and $4)$. This time, the shape of the flare's light curve is more symmetrical, with no time delay between the peaks of the $K_{\mathrm{s}}$ - and $L^{\prime}$-bands longer than $\sim 5 \mathrm{~min}$, the time resolution of the light curve. The peak fluxes of Flare B are $3.7 \pm 0.5$ and $8.2 \pm 0.5 \mathrm{mJy}$ (background-subtracted) in the $K_{\mathrm{s}}$ - and $L^{\prime}$-band, respectively.

No significant flare was recorded during the third night, which had an observed mean flux density of $\sim 4 \mathrm{mJy}$.

\subsection{X-rays}

No prominent X-ray flare ( $>10$ times the quiescent level) was detected in April 2009. We concentrate below on the significant peaks $(>3 \sigma)$ of the X-ray light curves detected in parallel to the NIR flares mentioned above.

Flare A coincided with a very little increase of the XMMNewton/EPIC cameras count rate (see Fig. 3, top and middle panels). Though barely significant $(3.5 \sigma)$, this could be the X-ray counterpart to Flare A and thus would be the faintest X-ray flare so far identified with XMM-Newton; all previous flares were at least a factor 2 brighter (Goldwurm et al. 2003; Porquet et al. 2003; Bélanger et al. 2005; Porquet et al. 2008). When considering the overall flare, the X-ray excess occurs in the middle 
Table 2. Properties of the X-ray/NIR flares observed so far.

\begin{tabular}{|c|c|c|c|c|c|c|c|}
\hline REFERENCES & Date & INSTRUMENTS & NIR FILTER ${ }^{a}$ & $L_{\mathrm{X}} / L_{\text {quies }}{ }^{b}$ & $\begin{array}{l}F_{\mathrm{NIR}}{ }^{c} \\
{[\mathrm{mJy}]}\end{array}$ & $\begin{array}{l}F_{\mathrm{NIR}}^{L^{\prime}{ }^{d}} \\
{[\mathrm{mJy}]}\end{array}$ & ID. \\
\hline$[1] \ldots \ldots$ & 2009 Apr. 1 & $X M M /$ VLT & $3.8 \mu \mathrm{m}\left(L^{\prime}\right)$ & $<7$ & 19 & $\ldots$ & $\mathrm{A}$ \\
\hline$[1] \ldots \ldots$ & 2009 Apr. 3 & $X M M / \mathrm{VLT}$ & $3.8 \mu \mathrm{m}\left(L^{\prime}\right)$ & 10 & 8 & & B \\
\hline$[2,3] \ldots$ & 2007 Apr. 4 & $X M M / \mathrm{VLT}$ & $3.8 \mu \mathrm{m}\left(L^{\prime}\right)$ & 103 & 26 & $\ldots$ & $\mathrm{C}$ \\
\hline$[2,4] \ldots$ & 2007 Apr. 4 & $X M M / \mathrm{HST}$ & $1.7 \mu \mathrm{m}$ & 26 & 7 & 16 & $\mathrm{D}$ \\
\hline$[2,4] \ldots$ & 2007 Apr. 4 & $X M M / \mathrm{HST}$ & $1.7 \mu \mathrm{m}$ & 37 & 4 & 9 & $\mathrm{E}$ \\
\hline$[2,4] \ldots$ & 2007 Apr. 2 & $X M M / \mathrm{HST}$ & $1.7 \mu \mathrm{m}$ & 14 & 10 & 22 & $\mathrm{~F}$ \\
\hline$[5,6] \ldots$ & 2004 Aug. 31 & $X M M / \mathrm{HST}$ & $1.6 \mu \mathrm{m}$ & $<40$ & 10 & 24 & $\mathrm{G}$ \\
\hline [7] ...... & 2005 Jul. 31 & Chandra/Keck & $3.8 \mu \mathrm{m}\left(L^{\prime}\right)$ & $<1.2$ & 15 & $\ldots$ & $\mathrm{H}$ \\
\hline$[7] \ldots \ldots$ & 2006 Jul. 17 & Chandra/Keck & $3.8 \mu \mathrm{m}\left(L^{\prime}\right)$ & 20 & $>5$ & $\ldots$ & I \\
\hline$[8] \ldots \ldots$ & 2005 Jun. 30 & Chandra/VLT & $2.1 \mu \mathrm{m}\left(K_{\mathrm{s}}\right)$ & 3 & 14 & 4 & $\mathrm{~J}$ \\
\hline [9] ...... & 2005 Jul. 7 & Chandra/VLT & $2.1 \mu \mathrm{m}\left(K_{\mathrm{s}}\right)$ & 15 & 11 & 3 & $\mathrm{~K}$ \\
\hline$[10] \ldots \ldots$ & 2003 Jun. 19 & Chandra/VLT & $2.1 \mu \mathrm{m}\left(K_{\mathrm{s}}\right)$ & 3 & $>7$ & $>2$ & $\mathrm{~L}$ \\
\hline
\end{tabular}

Notes. ${ }^{(a)}$ The HST 1.6 and $1.7 \mu$ m pass-bands correspond to the NICMOS filters $F 160 W$ and $F 170 M$, respectively. ${ }^{(b)}$ Peak X-ray luminosity $(2-10 \mathrm{keV})$ in units of the quiescent level $\left(\sim 2 \times 10^{33} \mathrm{erg} \mathrm{s}^{-1}\right.$, Baganoff et al. 2003). "> " and " $<$ " signs were added when the peak flux was missed or not simultaneous with the NIR peak. ${ }^{(c)}$ Background-subtracted peak NIR-fluxes in the bands indicated in the fourth column, dereddened assuming $A_{1.6 \mu \mathrm{m}}=4.5 \mathrm{mag}, A_{1.7 \mu \mathrm{m}}=5.03 \mathrm{mag}, A_{2.1 \mu \mathrm{m}}=2.8 \mathrm{mag}$, and $A_{3.8 \mu \mathrm{m}}=1.8 \mathrm{mag} .{ }^{(d)}$ Equivalent NIR flux in the $L^{\prime}$-band extrapolated assuming a power-law spectral index $\alpha=-1$ (see Sect. 4).

References. [1] This work, [2] Porquet et al. (2008), [3] Dodds-Eden et al. (2009), [4] Yusef-Zadeh et al. (2009), [5] Bélanger et al. (2005), [6] Yusef-Zadeh et al. (2006a), [7] Hornstein et al. (2007), [8] Eckart et al. (2008a), [9] Eckart et al. (2006a), [10] Eckart et al. (2004).

of the NIR flare, which is consistent with the flares that have been entirely covered in NIR and X-rays over the past (Eckart et al. 2006a, 2008a; Dodds-Eden et al. 2009). In view of the low statistics of this X-ray signal, we cannot extract proper spectral information. Assuming a typical photon spectral index, $\Gamma=2$ (with $\mathrm{d} N / \mathrm{d} E \propto E^{-\Gamma} \mathrm{ph} \mathrm{s}^{-1} \mathrm{~cm}^{-2} \mathrm{keV}^{-1}$ ), and a typical column density, $N_{\mathrm{H}}=10^{23} \mathrm{~cm}^{-2}$, we estimate through WebPIMMS that Flare A reached an unabsorbed, background-subtracted ${ }^{1}$, flux of $2.2 \times 10^{-12} \mathrm{erg} \mathrm{s}^{-1} \mathrm{~cm}^{-2}$ in the $2-10 \mathrm{keV}$ band. This translates into a peak luminosity of $1.7 \times 10^{34} \mathrm{erg} \mathrm{s}^{-1}$ at $8 \mathrm{kpc}$, i.e. $\sim 7$ times the quiscent level $\left(2.3 \times 10^{33} \mathrm{erg} \mathrm{s}^{-1}\right.$, Baganoff et al. 2003). This very low significance $X$-ray flare associated with a relatively bright NIR flare is quite surprising with regard to the bright X-ray flare $(\sim 100 \times$ the quiescence, Porquet et al. 2008$)$ detected simultaneously to a strong $L^{\prime}$ NIR flare $(\sim 30 \mathrm{mJy})$ in April 2007 (Dodds-Eden et al. 2009). It is however somewhat reminescent of the X-ray non-detection by the Chandra observatory of a $\sim 15 \mathrm{mJy} L^{\prime}$ flare observed in July 2005 by Hornstein et al. (2007). Yet, we stress that the point-spread functions (PSFs) and spectral responses of the Chandra/ACIS and $X M M-N e w t o n / E P I C$ instruments differ, which prevents straightforward comparisons. Indeed, that the EPIC's PSF is broader than ACIS's makes the XMM-Newton satellite less sensitive than Chandra to soft $(\Gamma \approx 2)$ and faint $X$-ray flares. The background subtracted peak XMM-Newton count rate of Flare A converts, indeed, to a Chandra/ACIS-I rate of $\sim 0.034$ counts s $\mathrm{s}^{-1}$ in the 2$8 \mathrm{keV}$ band. This would have lead to a clear Chandra detection, even more significant than two other faint X-ray/NIR flares observed with Chandra by Eckart et al. (2004, 2008a).

Flare B, on the other hand, was more significantly detected by XMM-Newton and observed to last longer ( 40 $\mathrm{min})$, in sync with the NIR light curve (see Fig. 4, top and middle panels). Using the same method as above, we estimate that its background-subtracted and unabsorbed peak flux is $3.2 \times$ $10^{-12} \mathrm{erg} \mathrm{s}^{-1} \mathrm{~cm}^{-2}$ in the $2-10 \mathrm{keV}$ band, i.e. $2.4 \times 10^{34} \mathrm{erg} \mathrm{s}^{-1}$ $(\sim 10 \times$ the quiescence). With Chandra it would have been detected at a rate of 0.049 counts $^{-1}(2-8 \mathrm{keV})$, which is

\footnotetext{
1 An average level of the quiescent count rate including the constant contibution of neighboring sources (Sect. 2.1) was removed as in Goldwurm et al. (2003).
}

still faint compared with the other X-ray flares with a known NIR counterpart as we discuss below. The fact that the NIR flux was relatively high about $100 \mathrm{~min}$ before Flare B with no simultaneous detectable X-ray emission is striking and could be another example of a "NIR-only flare" (Hornstein et al. 2007) or indicate that NIR flares can start earlier than the X-ray ones (Dodds-Eden et al. 2009).

Comparing Flare $\mathrm{A}$ and $\mathrm{B}$, it is manifest that the ratio of the peak NIR-flux over the peak X-ray flux is highly variable from one flare to another. It is known that the NIR-flare occurence rate is higher than the X-ray one, so it was expected that there should exist NIR flares with very little or even no detectable X-ray emission. But a somewhat counterintuitive fact that emerges now is that the brightest NIR flares are not necessarily accompanied by bright X-ray flares. Indications of that result had been obtained with the analysis of the July 2005 and July 2006 flares in Hornstein et al. (2007), although the peak NIR flux of the July 2005 event was missed. Similarly, Yusef-Zadeh et al. (2009) showed in their Figs. 21a and b with a consistent XMMNewton/HST dataset that the NIR-to-X-ray ratio fluctuates.

To allow better comparisons between flares concurrently observed in X-rays and NIR and visualize their disparities, we give an inventory of all the results published thus far in Table 2 and plot the X-ray peak fluxes vs. peak NIR-fluxes in Fig. 6. Despite a relatively easy comparison between the measurements conducted by XMM-Newton and Chandra, NIR comparisons between various authors are more difficult because of different observational setups and flux corrections (e.g., background removal, extinction correction). Flares A, B, and C (Table 2) are still easy to compare, because they were observed by the same instruments in the same pass-bands at roughly the same epoch. Flares $\mathrm{H}$ and $\mathrm{I}$ can also be added to this group, provided a background-correction is applied and consistent extinctions employed $^{2}$. Finally, the peak NIR-flux of the remaining seven flares (D, E, F, G, J, K, L) needs to be extrapolated to the $L^{\prime}$-band, assuming a particular infrared spectral index (see Sect. 4), to ensure consistency with the other flares.

\footnotetext{
2 We used an $L^{\prime}$ background of $3.6 \mathrm{mJy}$ as given in the caption of Fig. 5 and converted the extinctions quoted in the caption of Table 1 (Hornstein et al. 2007).
} 
G. Trap et al.: Concurrent X-ray, NIR, Sub-mm, and GeV $\gamma$-ray observations of Sgr A*

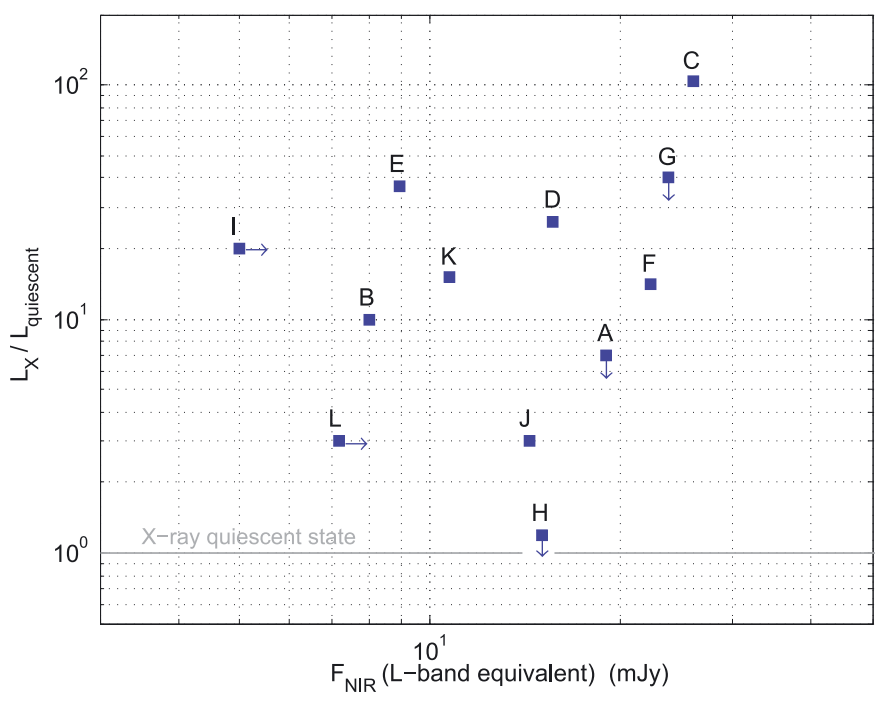

Fig. 6. Relation between the X-ray and NIR peak fluxes of all the flares so far covered with multi-wavelength observations. See Table 2 for details about each individual flare. Events A and B are the ones reported in this work. X-ray upper limits and NIR lower limits are indicated by down pointing and right pointing arrows, respectively.

As shown in Fig. 6, no particular correlation between the $\mathrm{X}$-ray and NIR strengths is apparent with the currently attainable statistics. Though the logarithmic scales of the plot axes do not reveal it, Flare $\mathrm{C}$ really stands out of the group as a strong event in both X-rays and NIR.

\subsection{Sub-mm}

On 2009 April 1, sub-mm observations overlapped with the NIR and X-ray coverages (Fig. 3). Sgr $\mathrm{A}^{\star}$ is found to be continuously variable at $870 \mu \mathrm{m}$, though on a longer time scale than at shorter wavelengths ${ }^{3}$. We first note that there are no abrupt changes in the APEX light curve at the time the VLT detects the rise of Flare A. This means that a simultaneous sub-mm counterpart to this NIR flare is not detected as such, and corroborates similar past non-detections (Marrone et al. 2008; Yusef-Zadeh et al. 2008; Eckart et al. 2008b).

$679 \pm 4$ min after 2009 April 1, 00:00:00 (UTC), the sub-mm flux of $\mathrm{Sgr} \mathrm{A}^{\star}$ peaked at $5.4 \pm 0.2 \mathrm{Jy}$, and marks the summit of a dramatic sub-mm flare, among the brightest ever detected, that lasted for $\sim 150 \mathrm{~min}$ and that we will refer to as Flare $\mathrm{A}^{\prime}$. When we subtract the background emission (taken as the minimum flux of the observation, $2.2 \mathrm{Jy}$ ), the peak is reduced to $3.2 \pm 0.2 \mathrm{mJy}$. The rise time of Flare $A^{\prime}$ is on the order of 20 min, which limits the size of the sub-mm flaring region to only $\sim 30 R_{\mathrm{S}}$ with the same argument as the one used in Sect. 3.1. Yet, this size constraint will probably be revised soon when Very Long Baseline Interferometry (VLBI) measurements are achieved in the sub$\mathrm{mm}$ range. Recent size measurements at $1.3 \mathrm{~mm}$ have indeed already yielded an intriguing upper limit to the quiescent size of Sgr $A^{\star}$ of $\sim 40 \mu$ as $\approx 4 R_{\mathrm{S}}$ (Doeleman et al. 2008).

Interestingly, Flare $\mathrm{A}^{\prime}$ lags Flare A by $\sim 200 \mathrm{~min}$ and is not associated with any other flaring episode, at least as far as we can tell from the X-ray light curve. As summarized in Table 3, this

\footnotetext{
3 Analogous variability, on timescales ranging from hours to days, was also reported at wavelengths longward of $\sim 1 \mathrm{~mm}$ (see e.g., Wright \& Backer 1993; Bower et al. 2002; Zhao et al. 2003; Herrnstein et al. 2004; Miyazaki et al. 2004; Mauerhan et al. 2005; Yusef-Zadeh et al. 2007).
}

Table 3. Short $\mathrm{mm}$ and sub-mm flare delays connected to X-ray or NIR flares.

\begin{tabular}{llcc}
\hline \hline References & Date & Instruments & $\begin{array}{c}\text { TimelaG }^{a} \\
{[\mathrm{~min}]}\end{array}$ \\
\hline$[1] \ldots \ldots$ & 2009 Apr. 1 & APEX $(870 \mu \mathrm{m})$ & 200 \\
{$[2,3,4] \ldots$} & 2006 Jul. 17 & SMA $(1.3 \mathrm{~mm}) / \mathrm{CSO}(850 \mu \mathrm{m})$ & 100 \\
{$[2] \ldots \ldots$} & 2005 Jul. 31 & SMA $(1.3 \mathrm{~mm})$ & 20 \\
{$[5] \ldots \ldots$} & 2008 Jun. 3 & APEX $(870 \mu \mathrm{m})$ & 90 \\
{$[6,7] \ldots \ldots$} & 2004 Jul. 7 & SMA $(890 \mu \mathrm{m})$ & $<120$ \\
{$[4] \ldots \ldots$} & 2007 Apr. 5 & SMA $(1.3 \mathrm{~mm})$ & 160 \\
{$[8] \ldots \ldots$} & 2004 Sep. 3 & CSO $(850 \mu \mathrm{m})$ & 160 \\
\hline
\end{tabular}

Notes. ${ }^{(a)}$ Time by which the sub-mm peak lags the X-ray or NIR peak. References. [1] This work, [2] Marrone et al. (2008), [3] Yusef-Zadeh et al. (2008), [4] Yusef-Zadeh et al. (2009), [5] Eckart et al. (2008b), [6] Eckart et al. (2006a), [7] Eckart et al. (2009), [8] Yusef-Zadeh et al. (2006a).

new flare detected with high confidence adds to a handful of past detections (sometimes yet debatable) of short $\mathrm{mm} / \mathrm{sub}-\mathrm{mm}$ activity in the aftermath of X-ray and/or NIR flares. One way often proposed to relate these phenomena is that X-ray/NIR flares are associated with a plasmoid expansion radiating via synchrotron emission (van der Laan 1966, see Sect. 5.1). If this plasmoid is also responsible for the production of X-rays through SSC emission, then as pointed out by Marrone et al. (2008), there is a tight relation connecting the timelag between the X-ray/NIR peak fluxes $\left(F_{\mathrm{X}}, F_{\mathrm{NIR}}\right)$ and the peak flux at a lower frequency $v$. This lag is given by

$$
\Delta t_{v} \propto\left(\frac{F_{\mathrm{X}}}{F_{\mathrm{NIR}}} B_{0}^{(p+2) / 2} v^{-(4+p) / 2}\right)^{1 / \delta(3+2 p)}
$$

where $B_{0}$ is the inital magnetic field, $p$ the spectral index of the radiating particles $\left(\mathrm{d} N / \mathrm{d} E \propto E^{-p}\right)$, and $\delta$ is a parameter depicting the speed at which the radius $R$ of the plasmoid grows $\left(R \propto t^{\delta}\right)$. So far, only three flares were fully covered by contemporaneous X-ray, NIR, and sub-mm observations and allow for a test of this relation: Flares A, H, and I. The comparison between $\mathrm{H}$ and I was done by Marrone et al. (2008), who showed a consistency between the data and the plasmoid/SSC picture.

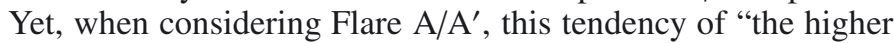
the ratio $F_{\mathrm{X}} / F_{\mathrm{NIR}}$, the longer the delay" no longer holds. Indeed, Flare I has a ratio about 10 times the ratio of Flare A, and still presents a delay about twice shorter. This tends to indicate that $\mathrm{X}$-ray/NIR flare and sub-mm flare emissions may not originate in the same particles (see also Sect. 5.1).

Regarding the other two APEX observing runs of April 2009, no sub-mm flares were detected, notwithstanding an obvious flux decrease observed during the last run. The minimum flux reached was $2.2 \mathrm{Jy}$, which justifies our previous choice for the steady quiescent background level.

\subsection{GeV $\gamma$-rays}

The Fermi light curves in the top panels of Figs. 3-5 are characterized by frequent data gaps on the order of $2 \mathrm{~h}$ during which the GC is outside of the LAT's FOV. The statistics within each bin is remarkably poor; many 10 min bins do not contain any photon at all. No significant peak in the light curves is detected.

Though Flare A and most of Flare $\mathrm{A}^{\prime}$ fell outside the coverage of the GC by the LAT, the rising flank of Flare B was 
coincident with the end of a LAT exposure. Zero photons were detected in the time bin at the peak of Flare B. Given the Poissonian statistics, the $3 \sigma$ (99.87\% confidence level) upper limit on the count rate of any source in the extraction region is 6.6 photons (Gehrels 1986) for the bin considered, which has an exposure of $6.2 \times 10^{4} \mathrm{~s}^{-1} \mathrm{~cm}^{-2}$. This converts to a flux upper limit for Flare B of $1.1 \times 10^{-4} \mathrm{ph} \mathrm{s}^{-1} \mathrm{~cm}^{-2}$, or $7.2 \times 10^{-8} \mathrm{erg} \mathrm{s}^{-1} \mathrm{~cm}^{-2}$, and a luminosity upper limit of $5.5 \times 10^{38} \mathrm{erg} \mathrm{s}^{-1}(0.1-200 \mathrm{GeV})$ at $8 \mathrm{kpc}$.

This is not very constraining with regard to the flux quoted for the GC Fermi central point source, 1FGL J1745.62900, in the first year catalog 4 (Abdo et al. 2010): $1.7 \times$ $10^{-6} \mathrm{ph} \mathrm{s}^{-1} \mathrm{~cm}^{-2}(0.1-100 \mathrm{GeV})$ for a photon spectral index of $\sim 2.2$. Fermi thus confirms the presence of a GeV source in the GC, as once detected by the Energetic GammaRay ExperimenT (EGRET) onboard the Compton Gamma-ray Observatory (Mayer-Hasselwander et al. 1998). The origin of 1FGL J1745.6-2900 is unknown. It is quite possible that this $\mathrm{GeV}$ emission is produced in GC molecular clouds just as the $\mathrm{TeV}$ emission of the same region (Ballantyne et al. 2007; Melia \& Fatuzzo 2011). But Sgr $A^{\star}$ itself is also of course a valid candidate, given the positional coincidence of the LAT source and the supermassive black hole: 1FGL J1745.6-2900 lies at the galactic coordinates $\left[l=359.941^{\circ}, b=-0.051^{\circ}\right]$ with a $95 \%$ confidence radius of $1.1^{\prime}$, which is only $\sim 21^{\prime \prime}$ away from $\mathrm{Sgr} \mathrm{A}^{\star}$. One way to unambiguously attribute this source to the supermassive black hole could have been the detection of a flaring activity correlated with the X-ray one. However, we did not detect any $\mathrm{GeV}$ signal with the sensitivity we achieved during Flare B (which limited our detection capability to large $\mathrm{GeV}$ flares of amplitude at least $\sim 65$ times the steady flux of 1FGL J1745.6-2900). The present study is hence analogous to the unfruitful previous attempts to discover correlated emission between the X-ray flares of Sgr $A^{\star}$ and the flux of the $\gamma$-ray sources IGR J17456-2901 and HESS J1745-290 (see Trap et al. 2010; Aharonian et al. 2008, for the INTEGRAL and HESS nondetections, respectively).

\section{NIR colors of Sgr $A^{\star}$}

Here we describe the results of the spectral imaging of $\mathrm{Sgr} \mathrm{A}^{\star}$ we made during the second night of VLT/NACO observations.

To compute the NIR photon spectral index $\alpha$, defined by $F_{v} \propto v^{\alpha}$, we produced the $L^{\prime}$ - and $K_{\mathrm{s}}$-band light curves of Sgr A ${ }^{\star}$ presented in Fig. 7 (top panel), for which we averaged the pairs of images taken at each filter switch. The error bars incorporate the standard deviation of these values as well as the error determined from the standard deviation of a comparison star of similar flux. Data points were interpolated to the same times and a large aperture was used in both bands to fully include the contribution of S 17. A global background correction (including $\mathrm{S} 17$ ) was made by subtracting the lowest flux value. It is important to note that $\alpha$ is highly dependent on the excess color $E\left(K_{\mathrm{s}}-L^{\prime}\right)=A_{K_{\mathrm{s}}}-A_{L^{\prime}}$, since

$$
\alpha=\frac{\log \left(10^{\left(A_{K_{\mathrm{s}}}-A_{L^{\prime}}\right) / 2.5} \times F_{K_{\mathrm{s}}}^{\mathrm{red}} / F_{L^{\prime}}^{\mathrm{red}}\right)}{\log \left(v_{K_{\mathrm{s}}} / v_{L^{\prime}}\right)},
$$

\footnotetext{
4 These measurements were obtained with a modeling of the underlying diffuse emission. A variability index of 18 was measured taking 11 time segments into account, which implies that 1FGL J1745.6-2900 is non-variable on a monthly timescale.
}
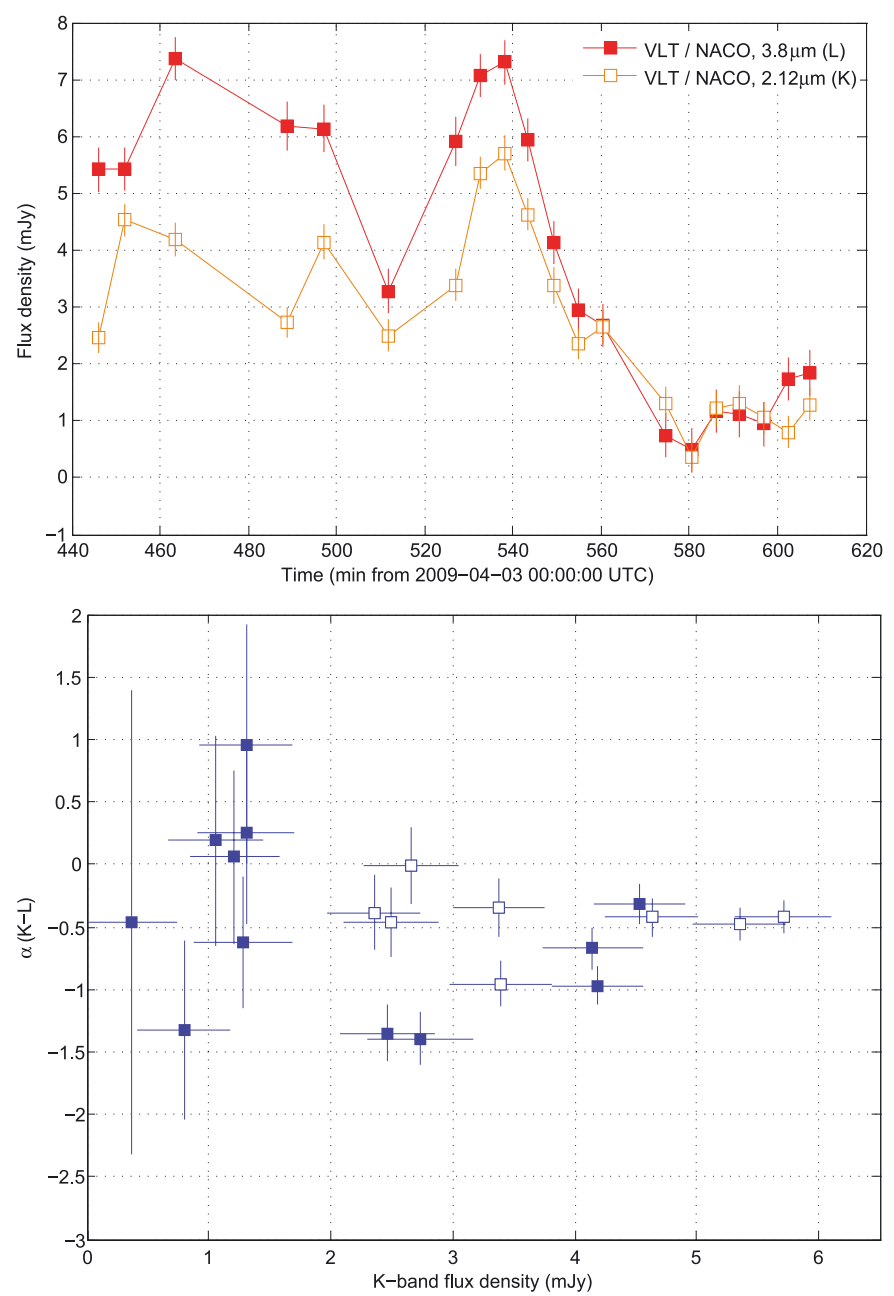

Fig. 7. (Top panel) NIR obervations of the second night backgroundsubtracted and corrected for extinction with $A_{L^{\prime}}=1.8 \mathrm{mag}$ and $A_{K_{\mathrm{s}}}=$ $A_{L^{\prime}}+E\left(K_{\mathrm{s}}-L^{\prime}\right)=3.3$ mag. (Bottom panel) Corresponding spectral index $\alpha$ plotted against dereddened $K_{\mathrm{s}}$-flux. Open squares indicate measurements made during Flare B.

where $F^{\text {red }}$ are the reddened fluxes. Using known early-type $\operatorname{stars}^{5}$ in the vicinity of Sgr A $\mathrm{A}^{\star}$, we determined an average color excess $E\left(K_{\mathrm{s}}-L^{\prime}\right)=1.5 \pm 0.1$ (see e.g. Hornstein et al. 2007). The corresponding values of $\alpha$ are plotted in Fig. 7 (bottom panel) as a function of the $K_{\mathrm{s}}$-band flux. Given the statistics of the data, no particular correlation between $\alpha$ and the flux is found. The mean value obtained over the duration of Flare B is $\alpha=-0.4 \pm 0.3$.

For comparison we list in Table 4 all previous attempts to measure the spectral indices of flares from $\operatorname{Sgr} A^{\star}$. The different values of $\alpha$ thus obtained are difficult to compare however, because each of these works either probed different spectral ranges/flux amplitudes/epochs or used different instruments/techniques/reddening laws/backgrounds. Owing to the experimental setup used in this study, our result can only be easily compared with the work of Hornstein et al. (2007). Likewise, these authors made use of a ground telescope with adaptive optics and the filter cycling technique, though they also accumulated data in the $H$ - and $M_{\mathrm{s}}$-bands, and used Keck's laser guide star. They found a constant "universal" value for the index of several flares, no matter the instantaneous flux, of

5 We used S 2, S 65, S 66, S 72, and S 87, which have $K$ magnitudes between 13.5-14.6 mag and are spectroscopically identified to have spectral types between O9-B2 (Eisenhauer et al. 2005). 
Table 4. Flare NIR spectral measurements.

\begin{tabular}{lclrc}
\hline \hline REFERENCES & Dates & INSTRUMENTS & BandS & $\alpha^{a}$ \\
& & & & \\
\hline$[1] \ldots \ldots$ & 2009 Apr. 3 & VLT/NACO & $2.1,3.8 \mu \mathrm{m}$ & $-0.4 \pm 0.3$ \\
{$[2] \ldots \ldots$} & 2005 Jul. 16, 31, 2006 May 2, Jul. 17 & Keck/NIRC2 & $1.6,2.1,3.8,4.7 \mu \mathrm{m}$ & $-0.6 \pm 0.2$ \\
{$[3] \ldots \ldots$} & 2007 Apr. 2, 5 & HST/NICMOS & $1.45,1.7 \mu \mathrm{m}$ & $-0.7 \pm 0.2,-1 \pm 0.3^{\dagger}$ \\
{$[4,5] \ldots$} & 2007 Apr. 4 & VLT/NACO, VISIR & $3.8,11.8 \mu \mathrm{m}$ & $>-1$ \\
{$[6] \ldots \ldots$} & 2005 Apr. 29 & Keck/OSIRIS & $2.02-2.38 \mu \mathrm{m}$ & $-2.6 \pm 0.9$ \\
{$[7] \ldots \ldots$} & 2005 Jun. 18 & VLT/SINFONI & $2-2.45 \mu \mathrm{m}$ & $-(0.6-1.3) \pm 0.2^{\dagger}$ \\
{$[8] \ldots \ldots$} & 2004 Jul. 15, 17 & VLT/SINFONI & $1.7-2.45 \mu \mathrm{m}$ & $-4 \pm 1$ \\
{$[9] \ldots \ldots$} & 2004 Jul. 26 & Keck/NIRC2 & $2.1,3.8 \mu \mathrm{m}$ & $-0.5 \pm 0.3$ \\
\hline
\end{tabular}

Notes. ${ }^{(a)} \alpha$ is defined by $F_{v} \propto v^{\alpha}$; ${ }^{\dagger}$ indicates when there is a correlation observed between the flux and the spectral index. In the case of Yusef-Zadeh et al. (2009), only the brightest flares are reported.

References. [1] This work, [2] Hornstein et al. (2007), [3] Yusef-Zadeh et al. (2009), [4] Dodds-Eden et al. (2009), [5] Trap et al. (2010), [6] Krabbe et al. (2006), [7] Gillessen et al. (2006), [8] Eisenhauer et al. (2005), [9] Ghez et al. (2005).

$\alpha=-0.6 \pm 0.2$, which is fully consistent with our result. Such a stable index seems to support a sort of a IR "quiescence" on average, even though this is debated (Dodds-Eden et al. 2010a).

Apparently all values for $\alpha$ recently obtained suggest $\alpha \gtrsim$ -1 , which translates to $\beta \gtrsim 0$ (with $v F_{v} \propto v^{\beta}$ ), thus justifying that "infrared flares are blue" and arise from a particle population distinct from the one producing the quiescent "sub-mm bump" (Dodds-Eden et al. 2009; Trap et al. 2010). Presuming a synchrotron origin for the NIR flares and the radiating electrons distributed in a power-law of index $p=1-2 \alpha\left(\mathrm{d} N / \mathrm{d} E \propto E^{-p}\right)$, this suggests that $p \lesssim 3$, which has important implications for the fit of flare spectra and in particular the SSC components (see Sect. 5.2).

\section{Discussion}

Below we discuss a simple modeling of Flare A/A' light curves and SED based on the expansion of a plasmoid releasing synchrotron and SSC radiation. Flare B SED is then analyzed in the context of different non-thermal emission processes. Models for SEDs are essentially static and only meant to show in which direction our data may constrain the theory of $\mathrm{Sgr}^{\star}$ 's flares. Evolutionary effects and the physical conditions leading to these scenarios will have to be studied in more detail in future works.

\subsection{Plasmoid expansion}

Over the past years, several authors have employed the adiabatic expansion of a plasmoid (van der Laan 1966) in the context of $\mathrm{cm}$ to sub-mm variability of Sgr A ${ }^{\star}$, often observed to follow X-ray/NIR flares (e.g., Yusef-Zadeh et al. 2006b; Eckart et al. 2006a, and the references listed in Table 3). The use of this expansion model for the flares of $\mathrm{Sgr} \mathrm{A}^{\star}$ is motivated by

- the timelags separating X-ray/NIR flares from sub-mm flares (Table 3);

- the timelags separating X-ray/NIR flares from radio flares (Yusef-Zadeh et al. 2009; Kunneriath et al. 2008);

- the timelags between different radio bands within the same flare (Yusef-Zadeh et al. 2006b) ${ }^{6}$;

- the increase of linear polarization as sub-mm flares reach their maximum (Marrone et al. 2008);

\footnotetext{
${ }^{6}$ Detailed models of these observations seem to exclude the simplest expansion scenario though, because the observed sizes of the radio emitting regions are bigger than the van der Laan predicted sizes (Falcke et al. 2009; Maitra et al. 2009).
}

- the similarity between the flare timescales in X-ray/NIR and $\mathrm{mm} / \mathrm{sub}-\mathrm{mm}$, which suggests that energy losses are not dominated by radiative cooling, with expansion a good alternative candidate ${ }^{7}$.

Figure 8 captures the essence of the model and gives the respective initial positions in the spectral domain of the wavebands used in this work. A ball of plasma of initial radius $R_{0}$, is filled with relavistic electrons of uniform volumic number density $n_{\mathrm{e}}$, arranged in a power-law $\left(\mathrm{d} n_{\mathrm{e}} / \mathrm{d} \gamma=k_{\mathrm{e}} \gamma^{-p}, \gamma_{\min } \leqslant \gamma \leqslant \gamma_{\max }\right)$. This plasmon is pervaded by a magnetic field, $B$, and yields an initial synchrotron spectrum made of two connected power-laws (Fig. 8, middle panel, red curve). Below the initial synchrotron self-absorption (SSA) frequency $v_{0}\left(\propto\left\{R_{0} k_{\mathrm{e}}\right\}^{2 /(p+4)} B^{(p+2) /(p+4)}\right.$, Gould 1979) at which the flux attains its maximal value, $F_{0}$, the spectrum is optically thick $\left(F_{v} \propto v^{5 / 2}\right)$. For $v>v_{0}$, the spectrum is optically thin $\left(F_{v} \propto v^{(1-p) / 2}\right)$ and breaks exponentially at a frequency proportional to $\gamma_{\max }^{2} B$. The dependence of the flux, $F_{v}$, on the radius, $R$, is given by

$F_{v}=F_{0}\left(\frac{v}{v_{0}}\right)^{5 / 2}\left(\frac{R}{R_{0}}\right)^{3} \frac{1-\exp \left(-\tau_{v}\right)}{1-\exp \left(-\tau_{0}\right)}$,

where the optical depth, $\tau_{v}$, obeys the equation

$\tau_{v}=\tau_{0}\left(\frac{v}{v_{0}}\right)^{-(p+4) / 2}\left(\frac{R}{R_{0}}\right)^{-(2 p+3)}$,

and where the optical depth $\tau_{0}$ at $v_{0}$ is set by the condition that $F_{v}$ be maximal $\left[\partial_{v} F_{v}\left(v_{0}, t\right)=0\right]$, or

$\exp \left(\tau_{0}\right)-\tau_{0}(p+4) / 5-1=0$.

In the following, we adopt a constant velocity, $V_{\exp }$, for the expansion so that

$R(t)=R_{0}+V_{\exp }\left(t-t_{0}\right)$.

To test this model with our multi-wavelength data of April 1, we propose two phenomenological scenarios (I and II) with different sets of values for the parameters $\left(p, R_{0}, v_{0}, F_{0}, V_{\exp }, t_{0}\right.$, see Table 5). To assess the viability of these models not only with the light curves but also spectrally, we calculate the plasmoid initial synchrotron spectra with particular values of $\gamma_{\min }, \gamma_{\max }$,

\footnotetext{
${ }^{7}$ Radiative cooling effects may however also affect the expansion model in the $\mathrm{mm}$ range ( $\mathrm{Li}$ et al. 2009).
} 

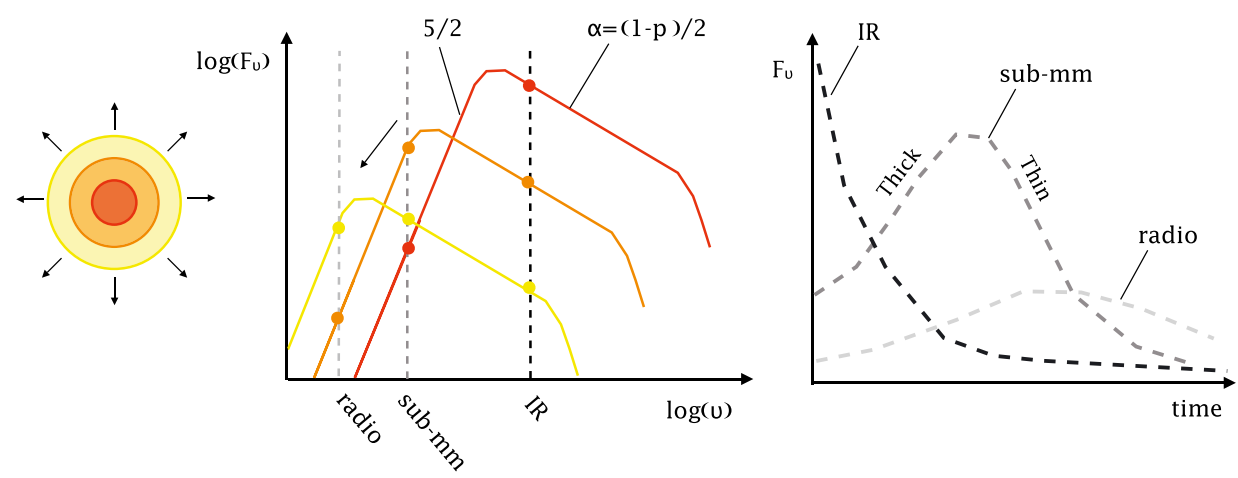

Fig. 8. Sketches of the van der Laan (1966) model. As the plasmoid expands (left panel), its initial self-absorbed synchrotron spectrum is shifted toward lower frequencies (middle panel). If the source is observed at optically thin frequencies from the beginning, then it remains optically thin and the flux keeps decreasing as time moves on (right panel, NIR curve). Alternatively, if the source is initially optically thick, then the flux rises until the source turns transparent and subsequently declines in the thin regime (right panel, sub-mm and radio curves).

Table 5. Parameters of Flare A and Flare B models.

\begin{tabular}{lccccccc}
\hline \hline & \multicolumn{2}{c}{ Flare A } & & \multicolumn{3}{c}{ Flare B } \\
\cline { 2 - 3 } \cline { 5 - 7 } Parameter & Model I & Model II & & SSC1 & SSC2 & SB \\
\hline$p \ldots \ldots \ldots$ & 5 & 2 & & 2 & 2.4 & $2.4-3.4$ \\
$R_{0}\left(R_{\mathrm{S}}\right) \ldots \ldots$ & 1 & 1 & & 1 & 1 & 1 \\
$v_{0}(\mathrm{~Hz}) \ldots \ldots$ & $2 \times 10^{12}$ & $2 \times 10^{12}$ & & - & - & - \\
$F_{0}(\mathrm{Jy}) \ldots \ldots$ & 22 & 17 & & - & - & - \\
$V_{\exp }(c) \ldots \ldots$ & 0.0017 & 0.005 & & - & - & - \\
$t_{0}(\min ) \ldots$ & 445 & 588 & & - & - & - \\
$\gamma_{\min } \ldots \ldots \ldots$ & 20 & 1 & & 1 & 1 & 1 \\
$\gamma_{\max } \ldots \ldots$ & $2 \times 10^{3}$ & 100 & & $1 \times 10^{4}$ & $2 \times 10^{4}$ & $6 \times 10^{5}$ \\
$B(\mathrm{G}) \ldots \ldots$ & 90 & 100 & & 2 & 5 & 21 \\
$n_{\mathrm{e}}\left(\mathrm{cm}^{-3}\right) \ldots$ & $1 \times 10^{9}$ & $3 \times 10^{9}$ & & $1 \times 10^{9}$ & $6 \times 10^{9}$ & $5 \times 10^{8}$ \\
\hline
\end{tabular}

$B$, and $n_{\mathrm{e}}$, and also consider the naturally associated SSC emission, using the formalism of Krawczynski et al. (2004) ${ }^{8}$. In all cases, the NIR emission is assumed to be optically thin, and so $v_{\text {submm }}<v_{0}<v_{\text {NIR }}$ (Fig. 8, middle panel). The NIR counterpart of Sgr $\mathrm{A}^{\star}$ is indeed known to be highly linearly polarized (Eckart et al. 2006b; Meyer et al. 2006a,b, 2007; Trippe et al. 2007) and, if it was optically thick, then an expansion model would anyhow predict a sub-mm peak flux lower than the NIR one (Fig. 8, right panel), which is inconsistent with the observations.

Model I. The scenario that requires the least number of ingredients to accomodate the NIR, sub-mm, and X-ray light data all at once is a plasmon initially producing the NIR and X-ray flare by synchrotron and SSC, respectively. When it expands, the NIR/X-ray flare decays and the sub-mm flare arises. Given the great difference between the NIR and sub-mm fluxes, this model demands an extremely steep power-law distribution $(p=5)$ and a high maximum flux $\left(F_{0}=22 \mathrm{Jy}\right)$ at the SSA turnover. We choose the different parameters to respect the flare size constraint $\left(R_{0}<10 R_{\mathrm{S}}\right)$ and the essential idea of the van der Laan (1966) model $\left(v_{\min }^{\text {syn }}<v_{0}\right.$, where $v_{\min }^{\text {sync }} \propto \gamma_{\min }^{2} v_{\mathrm{g}}, v_{\mathrm{g}}=e B /\left\{2 \pi m_{\mathrm{e}}\right\}$ is the gyration frequency, and $m_{\mathrm{e}}$ is the mass of the electron). Figure 9 (top and middle panels) shows that the overall NIR/sub-

\footnotetext{
${ }_{8}$ In the progress of this work, we found a problem in the treatment of the SSA in H. Krawczynski's code, which derived from a typo in the numerical expression in SI units of the absorption coefficient given by Longair (1994), which should be written: $\chi_{v}=20.9 \kappa^{\prime} B^{(p+2) / 2}(5.67 \times$ $\left.10^{8}\right)^{p} b(p) v^{-(p+4) / 2} \mathrm{~m}^{-1}$.
}

mm timescales and flux characteristics of Flare $\mathrm{A} / \mathrm{A}^{\prime}$ can be roughly reproduced (predictions at $100 \mu \mathrm{m}$ and $3 \mathrm{~mm}$ are also plotted), and also (bottom panel) that the observed initial SED constraints can be met. However, the initial steep NIR spectrum contradicts the measurements of $\alpha$ made with Flare B for example and exceeds the mid-infrared (MIR) upper limit obtained with VLT/VISIR during the April 2007 campaign (Trap et al. 2010 ) by a factor $\sim 4$. We therefore disfavor model I, although we cannot categorically exclude it given our lack of infrared spectral information on Flare A.

Model II. We now assume that Flare A and $\mathrm{A}^{\prime}$ are not directly connected by the same synchrotron power-law, and that Flare A simply acts as a trigger for a plasmoid expansion that will in time give rise to Flare $\mathrm{A}^{\prime}$. The plasmon power-law index can thus be chosen to be less steep $(p=2)$ and the couple of parameters $\left(\gamma_{\max }, B\right)$ so that the initial plasmoid spectrum breaks in the far-infrared (FIR). It thus gives no significant synchrotron emission in the NIR and X-ray bands, and the same holds for the SSC radiation. Yet, if the expansion starts at the beginning of the NIR flare, the relatively long time interval until the maximum of the sub-mm flare makes it difficult to obtain a narrow peak in the light curve at $870 \mu \mathrm{m}$. Therefore, we allow the time at which the expansion is initiated to be postponed by $140 \mathrm{~min}$, which gives an excellent fit to the APEX light curve (Fig. 10). This is our favored model, which could be tested with radio millimeter data, because it predicts a distinctive $3 \mathrm{~mm}$ flare peaking at $0.6 \mathrm{Jy}$ around $350 \mathrm{~min}$ after the onset of the NIR/X-ray flare. We stress that though ad hoc at this stage, this delayed expansion can be physically motivated in a "coronal mass ejection" picture because that it would take the accelerated particles $\sim 200 \mathrm{~min}$ to be advected out of the inner parts of the accretion flow, where the high viscosity would prevent an immediate adiabatic expansion (see Dodds-Eden et al. 2010b).

In all the above cases, we always used $R_{0}=R_{\mathrm{S}}$. But, given that Eqs. (3) and (4) only involve the ratio $R / R_{0}$, we would have obtained the exact same sub-mm light curves by multiplying $R_{0}$ by any factor $x$, provided the velocity $V_{\text {exp }}$ quoted in Table 5 was multiplied by the same $x$. Because $10 R_{\mathrm{S}}$ is an upper limit on the size of the flaring region, we find that the maximum expansion speed required is $0.05 c$, which is consistent with the other sub-relativistic expansions found previously (Yusef-Zadeh et al. 2008; Eckart et al. 2008b, 2009). This means, incidentally, that the plasmoid cannot escape the gravitational potential well of the black hole, unless it has a relativistic bulk motion, inside of a jet for example (Falcke et al. 2009; Maitra et al. 2009). 
G. Trap et al.: Concurrent X-ray, NIR, Sub-mm, and GeV $\gamma$-ray observations of Sgr A*
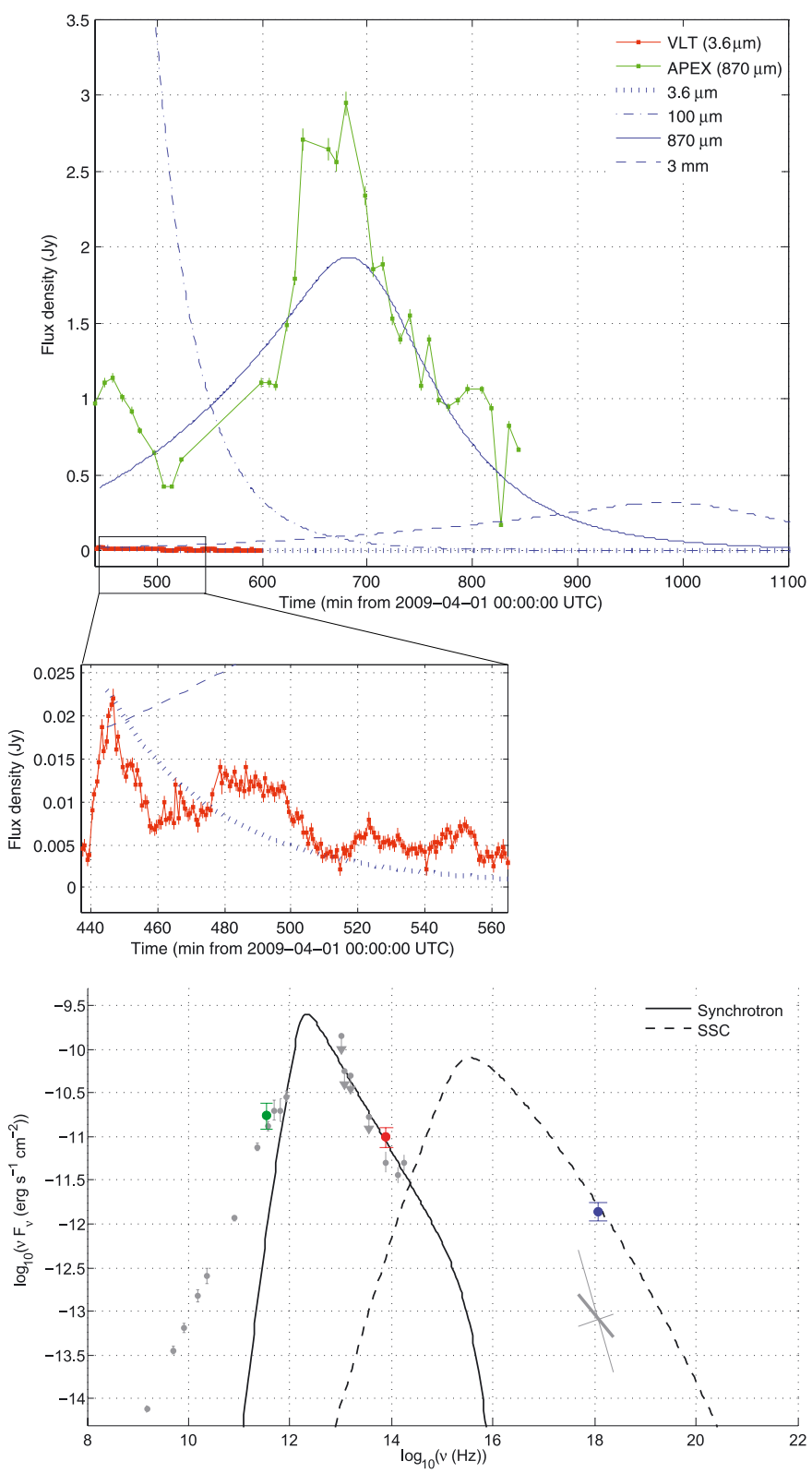

Fig. 9. (Top panel) APEX and VLT light curves plotted on the same scale. Theoretical predictions of model I for several wavelengths are overplotted in blue. (Middle panel) Magnified view of the VLT data. (Bottom panel) The synchrotron and SSC curves give the initial SED of the plasmoid in model I. They fit the simulaneous NIR and X-ray data (in red and blue respectively) of Flare A. The non-simultaneous sub$\mathrm{mm}$ data of Flare $\mathrm{A}^{\prime}$ are also indicated in green for information. The references of the quiescence data in light gray are given in Trap et al. (2010).

The modeling we present here compares well with previous works involving similar spectral windows (Eckart et al. 2008b, 2009), although we used only one plasmon compared with the multi-component models needed in these works. Nevertheless, our main result is that the simplest form of an abiabatic expansion scenario (model I) is inadequate to reproduce all our $\mathrm{NIR} /$ sub-mm/X-ray data at once, so that we favor expansion models in which NIR/X-ray flares are not initiated by the particles of the plasmon creating the sub-mm flare. This is in contrast with the study of Eckart et al. (2008b), in which models analogous to model I are generally favored. We note in particular that "model BI" of Eckart et al. (2008b) violates MIR upper

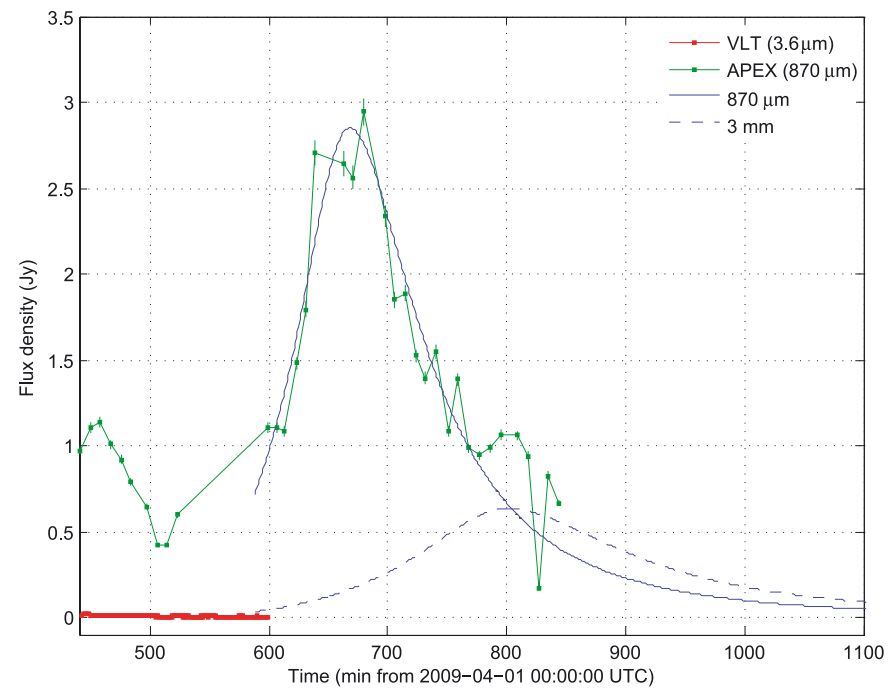

Fig. 10. Same as top panel of Fig. 9 for model II.

limits on Sgr A ${ }^{\star}$, and so does "model $\mathrm{AB} \alpha \phi 3$ " in Eckart et al. (2009), in spite of the NIR exponential break invoked to avoid the over-production of NIR flux.

We note also that a similar conclusion, disconnecting the NIR and radio portions of the spectrum in the frame of the van der Laan (1966) model, was reached by Mirabel et al. (1998) in the study of discrete ejections from the microquasar GRS $1915+105$.

Expansion models for the sub-mm flares of $\operatorname{Sgr} \mathrm{A}^{\star}$ should have two major telltale signatures: spectral indices in the sub$\mathrm{mm} / \mathrm{mm}$ range indicating an evolution from a thick to thin regime over the course of a flare, and high fluxes in the FIR (100-300 $\mu \mathrm{m}$, Fig. 9, top panel). The upcoming observations of the Galactic nucleus with Herschel and the Stratospheric Observatory for Infrared Astronomy (SOFIA) will perhaps allow the testing of these predictions.

\subsection{SED modeling}

Though sub-mm and NIR flares may be unrelated, NIR and $\mathrm{X}$-ray flares clearly derive from the same radiating particles given their simultaneity. Past theories of $\mathrm{Sgr} \mathrm{A}^{\star}$ flares usually invoked a combination of synchrotron and inverse Compton mechanisms (Markoff et al. 2001, 2007; Liu \& Melia 2002; Liu et al. 2004, 2006a,b; Yuan et al. 2003, 2004). We analyze here synchrotron and SSC emission for Flare B, for which we have NIR spectral information. This imposes a relatively hard electron spectrum ( $p \lesssim 3$, see Sect. 4) contrary to model I for Flare A, which had a softer spectrum $(p=5)$ as required by the expansion scenario. Because we do not have spectral information in X-rays, we propose three possible models (see Table 5 and Fig. 11) to represent the SED of this flare, each having an Xray slope either hard (SSC1), intermediate hard (SSC2), or soft (SB). In all three, the NIR flare is due to synchrotron emission. For SSC1 and SSC2, the X-ray flare results from SSC emission, which is the most commonly used configuration in the literature. Nonetheless, if the X-ray slope is soft, as for Flare \#2 in Porquet et al. (2008), then the SSC modeling is no longer valid, as it requires an enormous magnetic field strength on the order of $1000 \mathrm{G}$ (Dodds-Eden et al. 2009; Trap et al. 2010). In this case, a broadband synchrotron spectrum with a break (SB) due to radiative cooling could give rise to a soft X-ray flare (see DoddsEden et al. 2009, 2010b; Trap et al. 2010). 


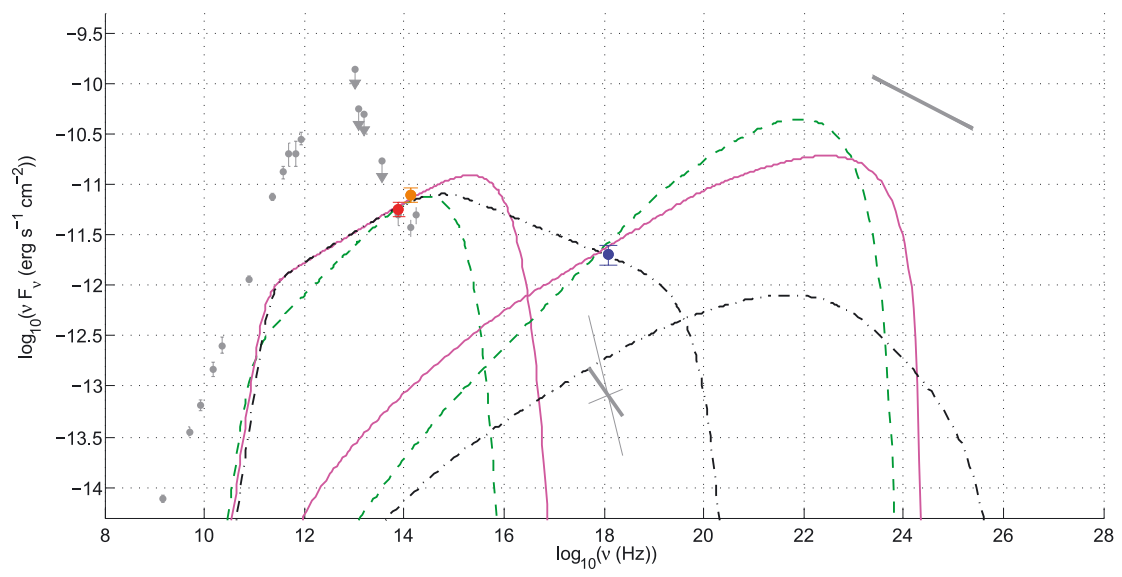

Fig. 11. SED of Flare B. $L^{\prime}-, K_{\mathrm{s}}$-band, and X-ray peak fluxes are plotted in red, orange, and blue, respectively. NIR error bars account for the uncertainty in the extinction correction. The 1-100 GeV spectrum of 1FGL J1745.6-2900 is indicated in dark gray. Models SSC1, SSC2, and SB correspond to the green dashed, magenta solid, black dash-dot lines, respectively.

We note in Fig. 11 that these flare spectra are all well below the 1-100 GeV spectrum of 1FGL J1745.6-2900, which is consistent with the non-detection reported in Sect. 3.4. In the progress of this work, we noticed a paper by Kusunose \& Takahara (2010) who model the time evolution of purely synchrotron (SB) scenarios for X-ray/NIR flares of Sgr A ${ }^{\star}$. The authors predict possible SSC counterparts observable with Fermi in a specific class of models, where the ratio of magnetic energy over particle energy is small. We have shown here that there was no detectable GeV excess during Flare B, but we stress that given our low statistics, we could only constrain a putative $\mathrm{GeV}$ flare to a flux lower than a few times $10^{-8} \mathrm{erg} \mathrm{s}^{-1} \mathrm{~cm}^{-2}$, whereas their "model C" induces a flux of only $\sim 2 \times 10^{-11} \mathrm{erg} \mathrm{s}^{-1} \mathrm{~cm}^{-2}$ at maximum. Except for an extraordinarily bright event, it is very likely that these models will not be testable with Fermi data.

\section{Conclusions}

Only a handful of flaring events have been observed simultaneously accross a broad range of wavelengths up to now. Here, we have reported the detection of two new X-ray/NIR flares, a strong sub-mm outburst possibly associated with one of them, and their $\mathrm{GeV} \gamma$-ray non-detection. These data allowed us to further characterize Sgr A ${ }^{\star}$ 's variability and test non-thermal radiative mechanisms usually employed to describe the physics of the flares.

One flare was followed at the VLT in two NIR spectral bands, thus demonstrating the feasibility of a filter-switching technique to measure the color of Sgr $A^{\star}$ with NACO. The resulting spectrum is found to be consistent with a power-law of index $\alpha=-0.4 \pm 0.3\left(F_{v} \propto v^{\alpha}\right)$, which is a "blue" spectrum $\left(\beta>0\right.$ with $\left.v F_{v} \propto v^{\beta}\right)$, implying that NIR flares arise from a particle population different from the one responsible for the quiescent sub-mm bump. For this flare, we could also check that there was no detectable in sync variation of the $\mathrm{GeV} \gamma$-ray GC source. The other NIR/X-ray flare was followed by a strong sub-mm flare, and was used to test the adiabatic expansion model, which is often used to account for the longer wavelength flare delays. In this context, we find it difficult to relate the NIR and sub$\mathrm{mm}$ events by the same single synchrotron plasmoid, because it would induce a too steep infrared spectrum with regard to other flares. Even assuming the NIR/X-ray event just acted as a trigger for the plasmon expansion, the long delay observed between the NIR/X-ray and sub-mm peaks spreads the theoretical light curve of the sub-mm flare too much to reproduce the data. However, we suggest an expanding plasmon could still drive the sub-mm flare light curve, provided it started expanding about a couple of hours after the X-ray/NIR event. These models can all be tested by radio observations obtained in April 2009 and predict a significant flux in the FIR that could be observed by the Herschel and SOFIA observatories in the near future. Hopefully, the source expansion scenario will also soon be put to test by VLBI direct size measurements in the sub-mm range (Doeleman et al. 2009). These studies of Sgr A ${ }^{\star}$ 's present variability, and those pursuing giant outbursts experienced by $\mathrm{Sgr} \mathrm{A}^{\star}$ in a recent past (Terrier et al. 2010; Ponti et al. 2010) should thus gradually bring an overall characterization of our Galactic nucleus' activity and help us put it into the general perspective of AGNs.

Acknowledgements. G.T. and K.D.E. warmly thank C. Lidman and G. Carraro for assistance with the NIR observations at Paranal, and the observers at the Chajnantor site (C. De Breuck, A. Lundgren, M. Dumke) for their efforts in planning and making the APEX obs. G.T. is also grateful to V. Reveret, F. Mattana, M. Sakano, and D. Marrone for enlightening discussions. This work has been partly supported by the french Agence Nationale pour la Recherche through grant ANR-06-JC-0047. G.P. acknowledges support via an EU Marie Curie Intra-European Fellowship under contract FP7-PEOPLE-2009-IEF-254279. The XMM-Newton project is an ESA Science Mission with instruments and contributions directly funded by ESA Member States and the USA (NASA). Parts of this study are based on observations made with ESO Telescopes at Paranal and Chajnantor Observatories under the programs LP 183.B-0100 and 69.A-0123.

\section{References}

Abdo, A. A., Ackermann, M., Ajello, M., et al. 2011, ApJ, submitted Aharonian, F., Akhperjanian, A. G., Aye, K.-M., et al. 2004, A\&A, 425, L13 Aharonian, F., Akhperjanian, A. G., Barres de Almeida, U., et al. 2008, A\&A, 492, L25

Atwood, W. B., Abdo, A. A., Ackermann, M., et al. 2009, ApJ, 697, 1071 Baganoff, F. K., Bautz, M. W., Brandt, W. N., et al. 2001, Nature, 413, 45 Baganoff, F., Maeda, Y., Morris, M., et al. 2003, ApJ 591, 891

Balick, B., \& Brown, R. 1974, ApJ, 194, 265

Ballantyne, D. R., Melia, F., Liu, S., \& Crocker, R. M. 2007, ApJ, 657, L13

Bélanger, G., Goldwurm, A., Goldoni, P., et al. 2004, ApJ, 601, L163

Bélanger, G., Goldwurm, A., Melia, F., et al. 2005, ApJ, 635, 1095

Bower, G. C., Falcke, H., Sault, R. J., \& Backer, D. C. 2002, ApJ, 571, 843

Dodds-Eden, K., Porquet, D., Trap, G., et al. 2009, ApJ, 698, 676

Dodds-Eden, K., Sharma, P., Quataert, E., et al. 2010, ApJ, 725, 450

Dodds-Eden, K., Gillessen, S., Fritz, T. K., et al. 2011, ApJ, 728, 37

Doeleman, S. S., Weintroub, J., Rogers, A. E. E., et al. 2008, Nature, 455, 78

Doeleman, S. S., Fish, V. L., Broderick, A. E., Loeb, A., \& Rogers, A. E. E. 2009, ApJ, 695, 59

Eckart, A., Baganoff, F. K., Morris, M., et al. 2004, A\&A, 427, 1

Eckart, A., Baganoff, F. K., Schödel, R., et al. 2006a, A\&A, 450, 535 
Eckart, A., Schödel, R, Meyer, L, et al, 2006b, A\&A, 455, 1

Eckart, A., Baganoff, F. K., Zamaninasab, M., et al. 2008a, A\&A, 479, 625

Eckart, A., Schödel, R., García-Marín, M., et al. 2008b, A\&A, 492, 337

Eckart, A., Baganoff, F. K., Morris, M. R., et al. 2009, A\&A, 500, 935

Eisenhauer, F., Genzel, R., Alexander, T., et al. 2005, ApJ, 628, 246

Falcke, H., Markoff, S., \& Bower, G. C. 2009, A\&A, 496, 77

Gehrels, N. 1986, ApJ, 303, 336

Genzel, R., Schödel, R., Ott, T., et al. 2003, Nature, 425, 934

Ghez, A., Wright, S., Matthews, K., et al. 2004, ApJ, 601, L159

Ghez, A. M., Hornstein, S. D., Lu, J. R., et al. 2005, ApJ, 635, 1087

Ghez, A. M., Salim, S., Weinberg, N. N., et al. 2008, ApJ, 689, 1044

Gillessen, S., Eisenhauer, F., Quataert, E., et al. 2006, ApJ, 640, L163

Gillessen, S., Eisenhauer, F., Trippe, S., et al. 2009, ApJ, 692, 1075

Goldwurm, A., Brion, E., Goldoni, P., et al. 2003, ApJ, 584, 751

Gould, R. J. 1979, A\&A, 76, 306

Güsten, R., Nyman, L. A., Schilke, P., et al. 2006, A\&A, 454, L13

Herrnstein, R. M., Zhao, J.-H., Bower, G. C., \& Goss, W. M. 2004, AJ, 127, 3399

Hornstein, S. D., Matthews, K., Ghez, A. M., et al. 2007, ApJ, 667, 900

Jansen, F., Lumb, D., Altieri, B., et al. 2001, A\&A, 365, L1

Kirsch, M. 2005, XMM-SOC-CAL-TN-0018: EPIC Status of Calibration and Data Analysis, Tech. Rep., XMM-Newton Science Operations Centre

Krabbe, A., Iserlohe, C., Larkin, J. E., et al. 2006, ApJ, 642, L145

Krawczynski, H., Hughes, S. B., Horan, D., et al. 2004, ApJ, 601, 151

Kunneriath, D., Eckart, A., Vogel, S., et al. 2008, J. Phys. Conf. Ser., 131, 012006

Kusunose, M., \& Takahara, F. 2011, ApJ, 726, 54

Lenzen, R., Hartung, M., Brandner, W., et al. 2003, in SPIE Conf. Ser. 4841, ed.

M. Iye, \& A. F. M. Moorwood, 944

Li, J., Shen, Z., Miyazaki, A., et al. 2009, ApJ, 700, 417

Liu, S., \& Melia, F. 2002, ApJ, 566, 77

Liu, S., Petrosian, V., \& Melia, F. 2004, ApJ, 611, L101

Liu, S., Melia, F., \& Petrosian, V. 2006a, ApJ, 636, 798

Liu, S., Petrosian, V., Melia, F., \& Fryer, C. L. 2006b, ApJ, 648, 1020

Longair, M. S. 1994, High Energy Astrophysics, Stars, the Galaxy and the interstellar medium (Cambridge, UK: Cambridge University Press), 2

Maitra, D., Markoff, S., \& Falcke, H. 2009, A\&A, 508, L13

Markoff, S., Falcke, H., Yuan, F., \& Biermann, P. 2001, A\&A, 379, L13

Markoff, S., Bower, G. C., \& Falcke, H. 2007, MNRAS, 379, 1519

Marrone, D. P., Baganoff, F. K., Morris, M. R., et al. 2008, ApJ, 682, 373

Mauerhan, J. C., Morris, M., Walter, F., \& Baganoff, F. K. 2005, ApJ, 623, L25

Mayer-Hasselwander, H., Bertsch, D., Dingus, B., et al. 1998, A\&A, 335, 161

Melia, F. 2007, The Galactic Supermassive Black Hole (Princeton, New Jersey: Princeton University Press)
Melia, F., \& Fatuzzo, M. 2011, MNRAS, 410, L23

Meyer, L., Eckart, A., Schödel, R., et al. 2006a, A\&A, 460, 15

Meyer, L., Schödel, R., Eckart, A., et al. 2006b, A\&A, 458, L25

Meyer, L., Schödel, R., Eckart, A., et al. 2007, A\&A, 473, 707

Mirabel, I. F., Dhawan, V., Chaty, S., et al. 1998, A\&A, 330, L9

Miyazaki, A., Tsutsumi, T., \& Tsuboi, M. 2004, ApJ, 611, L97

Muno, M., Baganoff, F., Bautz, M., et al. 2003, ApJ, 589, 225

Muno, M., Pfahl, E., Baganoff, F., et al. 2005, ApJ, 622, L113

Nishiyama, S., Tamura, M., Hatano, H., et al. 2009, ApJ, 702, L56

Pierce-Price, D., Richer, J. S., Greaves, J. S., et al. 2000, ApJ, 545, L121

Ponti, G., Trap, G., Goldwurm, A., et al. 2009, The Astronomer's Telegram, 2038, 1

Ponti, G., Terrier, R., Goldwurm, A., Belanger, G., \& Trap, G. 2010, ApJ, 714 732

Porquet, D., Predehl, P., Aschenbach, B., et al. 2003, A\&A, 407, L17

Porquet, D., Grosso, N., Predehl, P., et al. 2008, A\&A, 488, 549

Reid, M. J., Menten, K. M., Zheng, X. W., Brunthaler, A., \& Xu, Y. 2009, ApJ, 705,1548

Rousset, G., Lacombe, F., Puget, P., et al. 2003, in SPIE Conf. Ser. 4839, ed. P. L. Wizinowich, \& D. Bonaccini, 140

Sakano, M., Warwick, R., Decourchelle, A., \& Predehl, P. 2004, MNRAS, 350, 129

Siringo, G., Kreysa, E., Kovács, A., et al. 2009, A\&A, 497, 945

Strüder, L., Briel, U., Dennerl, K., et al. 2001, A\&A, 365, L18

Terrier, R., Ponti, G., Bélanger, G., et al. 2010, ApJ, 719, 143

Trap, G., Goldwurm, A., Terrier, R., et al. 2010, Adv. Space Res., 45, 507

Trippe, S., Paumard, T., Ott, T., et al. 2007, MNRAS, 375, 764

Turner, M. J. L., Abbey, A., Arnaud, M., et al. 2001, A\&A, 365, L27

van der Laan, H. 1966, Nature, 211, 1131

Viehmann, T., Eckart, A., Schödel, R., et al. 2005, A\&A, 433, 117

Wang, Q. D., Lu, F. J., \& Gotthelf, E. V. 2006, MNRAS, 367, 937

Wright, M. C. H., \& Backer, D. C. 1993, ApJ, 417, 560

Yuan, F., Quataert, E., \& Narayan, R. 2003, ApJ, 598, 301

Yuan, F., Quataert, E., \& Narayan, R. 2004, ApJ, 606, 894

Yusef-Zadeh, F., Bushouse, H., Dowell, C. D., et al. 2006a, ApJ, 644, 198

Yusef-Zadeh, F., Roberts, D., Wardle, M., Heinke, C. O., \& Bower, G. C. 2006b, ApJ, 650, 189

Yusef-Zadeh, F., Wardle, M., Cotton, W. D., Heinke, C. O., \& Roberts, D. A 2007, ApJ, 668, L47

Yusef-Zadeh, F., Wardle, M., Heinke, C., et al. 2008, ApJ, 682, 361

Yusef-Zadeh, F., Bushouse, H., Wardle, M., et al. 2009, ApJ, 706, 348

Zhao, J.-H., Young, K. H., Herrnstein, R. M., et al. 2003, ApJ, 586, L29

Zylka, R., \& Mezger, P. G. 1988, A\&A, 190, L25 